\title{
New species of Pseudosperma (Agaricales, Inocybaceae) from Pakistan revealed by morphology and multi-locus phylogenetic reconstruction
}

\author{
Malka Saba', Danny Haelewaters ${ }^{2,3,4}$, Donald H. Pfister ${ }^{2}$, Abdul Nasir Khalid ${ }^{5}$ \\ I Department of Plant Sciences, Quaid-i-Azam University, Islamabad, 45320, Pakistan 2 Farlow Herbarium \\ of Cryptogamic Botany, Harvard University, Cambridge, Massachusetts, USA 3 Department of Botany and \\ Plant Pathology, Purdue University, West Lafayette, Indiana, USA 4 Faculty of Science, University of South \\ Bohemia, Čské Budějovice, Czech Republic 5 Department of Botany, University of the Punjab, Lahore, Pakistan \\ Corresponding author: Malka Saba (rustflora@gmail.com; msaba@qau.edu.pk)
}

Academic editor: Olivier Raspé | Received 3 February 2019 | Accepted 12 May 2020 | Published 10 July 2020

Citation: Saba M, Haelewaters D, Pfister DH, Khalid AN (2020) New species of Pseudosperma (Agaricales, Inocybaceae) from Pakistan revealed by morphology and multi-locus phylogenetic reconstruction. MycoKeys 69: 1-31. https://doi.org/10.3897/mycokeys.69.33563

\begin{abstract}
During fungal surveys between 2012 and 2014 in pine-dominated forests of the western Himalayas in Pakistan, several collections of Pseudosperma (Agaricales, Inocybaceae) were made. These were documented, based on morphological and molecular data. During this work, three new species came to light, which are here formally described as Pseudosperma brunneoumbonatum, P. pinophilum and P. triacicularis. These species belong in the genus Pseudosperma fide Matheny et al. (2019) = Pseudosperma clade fide Matheny $(2005)$ = Inocybe sect. Rimosae s.s. fide Larsson et al. (2009). Macro- and micro-morphological descriptions, illustrations and molecular phylogenetic reconstructions of the studied taxa are provided. The new species are differentiated from their close relatives by basidiospore size and colouration of basidiomata. Molecular phylogenetic relationships are inferred using ITS (ITS1-5.8S-ITS2), nrLSU and mtSSU sequence data. All three newly-described taxa likely share an ectomycorrhizal association with trees in the genus Pinus. In addition, five names are recombined in Inosperma, Mallocybe and Pseudosperma. These are Inosperma vinaceobrunneum, Mallocybe erratum, Pseudosperma alboflavellum, Pseudosperma friabile and Pseudosperma neglectum.
\end{abstract}

\section{Keywords}

Ectomycorrhizal fungi, molecular systematics, phylogeny, Pinus roxburghii, southern Asia, taxonomy

Copyright Malka Saba et al. This is an open access article distributed under the terms of the Creative Commons Attribution License (CC BY 4.0), which permits unrestricted use, distribution, and reproduction in any medium, provided the original author and source are credited. 


\section{Introduction}

Inocybe (Fr.) Fr. (Agaricales, Inocybaceae) in the broad sense (sensu lato) is a highly diverse, ectomycorrhizal genus comprising about 735 known species worldwide (Ullah et al. 2018). Inocybe has a widespread distribution and is found commonly in temperate areas and, to a lesser extent, in the tropics (Matheny et al. 2009, Bougher et al. 2012, Matheny et al. 2012). Multi-locus phylogenies of the Inocybaceae by Matheny et al. $(2002,2009)$ and Matheny $(2005)$ have confirmed that the family is monophyletic. Matheny $(2005,2009)$ recognised seven major clades within the Inocybaceae; clade names were given with a suggestion to recognise each informally at the generic rank within the family.

Inocybe section Rimosae sensu stricto (fide Larsson et al. 2009, = clade Pseudosperma fide Matheny 2005), traditionally placed in subgenus Inosperma (Kuyper 1986, Kobayashi 2002), is one of the seven major clades in the Inocybaceae. Species of this clade are typically characterised by a rimose pileus surface; furfuraceous to furfuraceous-fibrillose stipe; absence of metuloids and pleurocystidia; smooth, elliptical to indistinctly phaseoliform basidiospores; and cylindrical to clavate cheilocystidia. Unlike species in clades Mallocybe and Inosperma (fide Matheny 2005) and the genera Auritella Matheny \& Bougher and Tubariomyces Esteve-Rav. \& Matheny, all of which also lack pleurocystidia, the basidia of species in the Pseudosperma clade are hyaline and not necropigmented. The Nothocybe clade is represented by only one species, $I$. distincta K.P.D. Latha \& Manim. This species also lacks pleurocystidia and can be differentiated based on molecular phylogenetic data (Latha et al. 2016). Some lineages in the Pseudosperma clade are composed of multiple cryptic species (Ryberg et al. 2008) and they form ectomycorrhizal associations with a broad range of host trees, both gymnosperms and angiosperms (Kuyper 1986, Stangl 1989, Jacobsson 2008).

Based on a six-locus phylogeny of the family Inocybaceae, Matheny et al. (2019) formally proposed genus names for the different clades: Inocybe sensu stricto, Inosperma (Kühner) Matheny \& Esteve-Rav. (elevated from subgenus-level), Mallocybe (Kuyper) Matheny, Vizzini \& Esteve-Rav. (elevated from subgenus-level), Nothocybe Matheny \& K.P.D. Latha and Pseudosperma Matheny \& Esteve-Rav., in addition to Auritella and Tubariomyces that were previously described. The authors decided to provide a formal generic system to name the different clades, because this allows better communication and provides the taxonomic precision needed for conservation issues and identification of biodiversity hot spots.

During an investigation of ectomycorrhizal fungi associated with pine species in Pakistan, three species of Pseudosperma with affiliation to sect. Rimosae s.s. were collected in the vicinity of pure stands of Pinus roxburghii Sarg. and P. wallichiana A.B. Jacks. The species were documented, based on morphological and molecular phylogenetic data. In this paper, we describe these taxa as new species, P. brunneoumbonatum, $P$. pinophilum and P. triaciculare. This is the first study in which a combination of morphological and multi-locus phylogenetic data was used to describe species of Inocybe sensu lato in sect. Rimosae s.s. - now genus Pseudosperma - from Pakistan. 


\section{Material and methods}

\section{Morphological studies}

Basidiomata were collected, described and photographed in the field. Colours were compared to the Munsell Soil Color Charts (1975) guide. Collections were dried using a food dehydrator (at $39{ }^{\circ} \mathrm{C}$ for $7-9$ hours). Microscopic characters were observed in the laboratory using hand-cut sections of basidiomata mounted in a 5\% aqueous solution of potassium hydroxide $(\mathrm{KOH})$ and in Congo red. Micromorphological analysis, photographs and measurements were made, using an Olympus BX40 light microscope with Olympus XC50 digital camera and Microsuite special edition software 3.1 (Soft imaging solutions $\mathrm{GmbH}$ ). Thirty basidiospores were measured from each collection cited. Measurements include the range with extremes provided in parentheses. Q values (length/width ratios) and mean values (average basidiospore length and width) are also provided. Line drawings were made with a Leitz camera Lucida (Wetzlar, Germany). Collections of the newly-described species are deposited at LAH (University of the Punjab Herbarium, Lahore) and FH (Farlow Herbarium, Harvard University).

\section{DNA extraction, PCR amplification and DNA sequencing}

Genomic DNA was extracted from a $20 \mathrm{mg}$ piece of dried tissue by a modified CTAB method (Lee et al. 1988). Loci examined during this study include the complete ITS region (ITS1-5.8S-ITS2) of the nuclear ribosomal RNA gene (hereafter ITS), the first ca. $900 \mathrm{bp}$ of the nuclear $28 \mathrm{~S}$ rRNA gene (nrLSU) and the mitochondrial small subunit rRNA gene (mtSSU).

Primers used for amplification were: ITS1F (Gardes and Bruns 1993) and ITS4 (White et al. 1990) for ITS; LR0R and LR5 for nrLSU (Vilgalys and Hester 1990); and MS1 and MS2 for mtSSU (White et al. 1990). The amplification reaction mixture contained $2.5 \mu \mathrm{l}$ Econo buffer, $0.5 \mu \mathrm{l}$ dNTPs, $1.25 \mu \mathrm{l}$ each primer, $0.125 \mu \mathrm{l}$ Econo Taq, $14.375 \mu \mathrm{l}$ of deionised water and $5 \mu \mathrm{l}$ of template DNA. Thermal profile of PCR for ITS was initial denaturation at $94^{\circ} \mathrm{C}$ for 1 min.; then 35 cycles of denaturation at $94^{\circ} \mathrm{C}$ for 1 min, annealing at $53^{\circ} \mathrm{C}$ for $1 \mathrm{~min}$ and extension at $72^{\circ} \mathrm{C}$ for $1 \mathrm{~min}$; and final extension at $72^{\circ} \mathrm{C}$ for $8 \mathrm{~min}$. For nrLSU: $94^{\circ} \mathrm{C}$ for $2 \mathrm{~min}$; then 40 cycles of $94^{\circ} \mathrm{C}$ for $1 \mathrm{~min}, 52^{\circ} \mathrm{C}$ for $1 \mathrm{~min}$ and $72{ }^{\circ} \mathrm{C}$ for $1: 30 \mathrm{~min}$; and $72^{\circ} \mathrm{C}$ for $5 \mathrm{~min}$. For $\mathrm{mtSSU}: 95^{\circ} \mathrm{C}$ for $10 \mathrm{~min}$; then 30 cycles of $95^{\circ} \mathrm{C}$ for $30 \mathrm{sec}, 52^{\circ} \mathrm{C}$ for $30 \mathrm{sec}$ and $72{ }^{\circ} \mathrm{C}$ for $40 \mathrm{sec}$; and $72{ }^{\circ} \mathrm{C}$ for $7 \mathrm{~min}$.

PCR products were run on $1 \%$ agarose gel, stained with ethidium bromide and bands were visualised under a UV transilluminator. Amplified PCR products of the ITS region were sent for purification and bidirectional sequencing to Macrogen (Republic of Korea). PCR products of $28 \mathrm{~S}$ and $16 S$ were purified using QIAquick PCR purification kit (Qiagen, Stanford, California) as per manufacturer's guidelines and sequencing reactions were performed using the Big Dye Terminator v3.1 Cycle Kit (Life Technologies, Carlsbad, California). Sequencing was carried out using the same primers as those used for PCR. 


\section{Sequence alignment and phylogenetic analysis}

Sequences were manually edited and assembled in BioEdit v7.2.6 (Hall 1999). Generated ITS sequences were trimmed with the conserved motifs 5'-CATTA- and -GACCT-3' (Dentinger et al. 2011) and the alignment portion between these motifs was included in subsequent analyses. BLASTn searches were performed in NCBI GenBank. Three data matrices for phylogenetic inferences were prepared: a concatenated ITS-nrLSU-mtSSU dataset of Rimosae s.s. and Inosperma clades (dataset \#1); a concatenated ITS-nrLSU$\mathrm{mtSSU}$ dataset of Rimosae s.s. subclade A (dataset \#2); and an extended nrLSU dataset of Rimosae s.s. subclade A (dataset \#3). We applied the clade names used by Larsson et al. (2009) in the methods and results sections to maintain consistency and clarity.

Sequences were downloaded from NCBI GenBank (https://www.ncbi.nlm.nih. gov/genbank/). The majority of sequences were generated in the studies of Larsson et al. (2009) and Ryberg et al. (2008), complemented by nrLSU sequences from more recent papers and our newly-generated sequences (details and references in Table 1). Sequences were aligned by locus (ITS+nrLSU, mtSSU) using Muscle v3.7 (Edgar 2004), available in the Cipres Science Gateway (Miller et al. 2010). Ambiguously-aligned regions were detected and removed using trimAl v1.3 (Capella-Gutiérrez et al. 2009), with the following parameters: $60 \%$ gap threshold, $50 \%$ minimal coverage. The ITS1, $5.8 S$, ITS2 and nrLSU loci were extracted from the aligned ITS +nrLSU dataset. This allowed us to select substitution models for each region, which is important because there are different rates of evolution within and amongst these components and rDNA loci (e.g. Hillis and Dixon 1991, discussion in Haelewaters et al. 2018).

The data for each locus were concatenated in MEGA7 (Kumar et al. 2016) to create matrices of $2537 \mathrm{bp}$ with sequence data for 123 isolates in the Rimosae s.s. and Inosperma dataset (\#1); and of 2561 bp for 50 isolates in the Rimosae s.s. subclade A dataset (\#2). The nrLSU dataset (\#3) consisted of $1383 \mathrm{bp}$ for 62 isolates belonging to Rimosae s.s. subclade A. Alignments generated during this study are available for download in NEXUS format from the figshare online repository (https://doi.org/10.6084/ m9.figshare.c.4701338). Nucleotide substitution models were selected for each locus (ITS1, 5.8S, ITS2, nrLSU, mtSSU) using jModelTest2 (Darriba et al. 2012) by considering the Akaike Information Criterion (AIC). For both concatenated datasets \#1 and \#2, models were selected for ITS1, 5.8S, ITS2, nrLSU and mtSSU; for dataset \#3, the best model was selected for nrLSU. Maximum likelihood was inferred for each dataset under partitioned models using IQ-tree (Nguyen et al. 2015, Chernomor et al. 2016). Ultrafast bootstrapping was done with 1000 replicates (Hoang et al. 2017).

\section{Results}

Nucleotide alignment datasets and phylogenetic inferences

Concatenated dataset \#1 consisted of 2537 characters, of which 1448 were constant and 841 were parsimony-informative. A total of 123 isolates were included, of which 
Naucoria bohemica Velen., N. salicis P.D. Orton and N. submelinoides (Kühner) Maire (Agaricales, Hymenogastraceae) served as outgroup taxa. The following models were selected by jModelTest2 (AIC): TIM2+I+G (ITS1, $-\operatorname{lnL}=6194.8143)$, TPM2+I (5.8S, $-\operatorname{lnL}=445.7026), \mathrm{GTR}+\mathrm{G}(\mathrm{ITS} 2,-\operatorname{lnL}=4445.9240), \mathrm{TIM} 3+\mathrm{I}+\mathrm{G}(\mathrm{nrLSU},-\operatorname{lnL}=$ $10227.1599)$ and $\mathrm{TVM}+\mathrm{I}+\mathrm{G}(\mathrm{mtSSU},-\operatorname{lnL}=4034.3342)$. Concatenated dataset \#2 consisted of 2561 characters, of which 2026 were constant and 399 were parsimonyinformative. A total of 50 isolates were included, of which P. obsoletum (Romagn.) Matheny \& Esteve-Rav. and P. perlatum (Cooke) Matheny \& EsteveRav. (Rimosae s.s. subclade B, fide Larsson et al. 2009) served as outgroup taxa. The following models were selected by jModelTest2 (AIC): TPM2uftG (ITS1, $-\operatorname{lnL}=2070.5127)$, TrNef (5.8S, $-\ln L=261.9437), T P M 1 u f+I+G(I T S 2,-\ln L=1683.9167), T r N+I+G(n r L S U$, $-\operatorname{lnL}=4608.2667)$ and TIM2 $+\mathrm{G}(\mathrm{mtSSU},-\operatorname{lnL}=1758.7165)$. Finally, dataset \#3 consisted of 1383 characters, of which 1091 were constant and 205 were parsimonyinformative. A total of 67 isolates were included, again with $N$. bohemica, $N$. salicis and $N$. submelinoides as outgroup taxa. For this single-locus dataset, the $\mathrm{Tr}+\mathrm{I}+\mathrm{G}$ model gave the best-scoring tree ( $\mathrm{nrLSU},-\operatorname{lnL}=5708.4547)$.

Six strongly supported clades (referred to as subclades A to F, fide Larsson et al. 2009) and two additional clades with maximum support were recovered in the ML analysis of the Rimosae s.s. and Inosperma clades (dataset \#1, Figure 1). A strongly supported clade with 35 sequences corresponds with Rimosae s.s. subclade A and includes the following species: P. bulbosissimum (Kühner) Matheny \& Esteve-Rav., P. melliolens (Kühner) Matheny \& Esteve-Rav., P. pinophilum sp. nov., P. rimosum (Bull.) Matheny $\&$ Esteve-Rav. (s.s.), P. sororium (Kauffman) Matheny \& Esteve-Rav. and P. umbrinellum (Bres.) Matheny \& Esteve-Rav. In addition, numerous taxa on single branches and less-supported clades are recovered.

In all three phylogenetic reconstructions (Figures 1-3), there is high support (BS $=81-100$ ) for the grouping of $P$. pinophilum sp. nov. with $P$. cf. rimosum from Europe (isolates JV8125 and PC080925). This clade is deeply nested in Rimosae s.s. subclade A (fide Larsson et al. 2009). Pseudosperma brunneoumbonatum sp. nov. is retrieved as sister to an undescribed species from Papua New Guinea (isolates TR104_05 and TR133_05) with high support $(B S=96-100)$. In both datasets $\# 2$ and \#3, this clade, again, is deeply nested in Rimosae s.s. subclade A. In dataset \#1, however, the clade P. brunneoumbonatum - I. sp. Papua New Guinea is placed between Rimosae subclades A and B (fide Larsson et al. 2009) with maximum support (Figure 1). Pseudosperma triaciculare sp. nov. is retrieved with high support (BS $=95-100)$ as an independent clade without clear affinities outside of Rimosae s.s. subclade A.

Our phylogenetic reconstructions (Figures 1-3) indicate that several undescribed species occur in Rimosae s.s. subclade A (see Discussion). All ML analyses recovered two new Pakistani species, $P$. triaciculare and $P$. pinophilum, as strongly-supported lineages nested within this subclade, whereas a third species, P. brunneoumbonatum, forms a strongly-supported clade outside of what is currently recognised as subclade A. These three new taxa from Pakistan can be distinguished, based on molecular phylogenetic data, as well as morphology and ecology. 


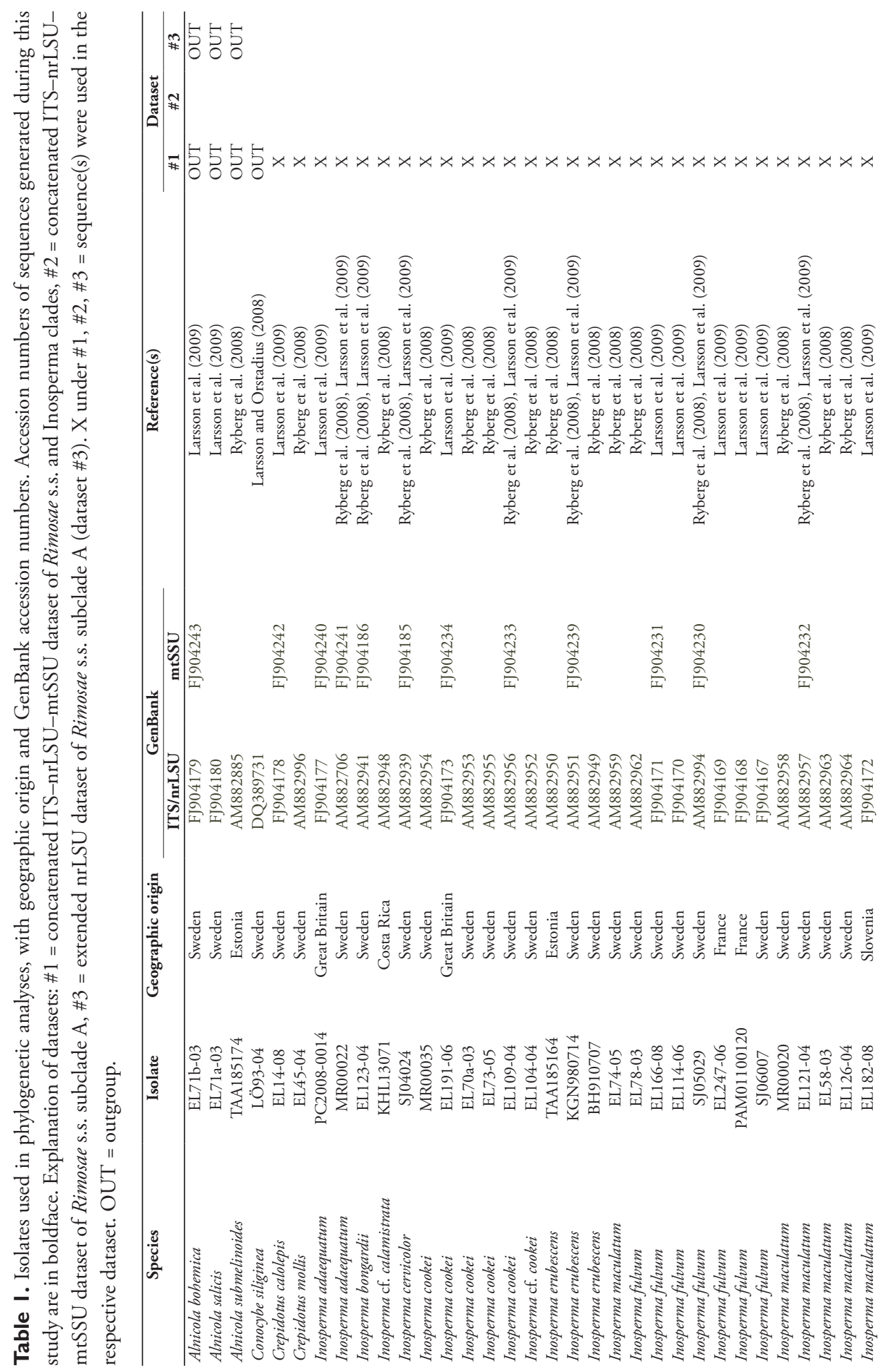




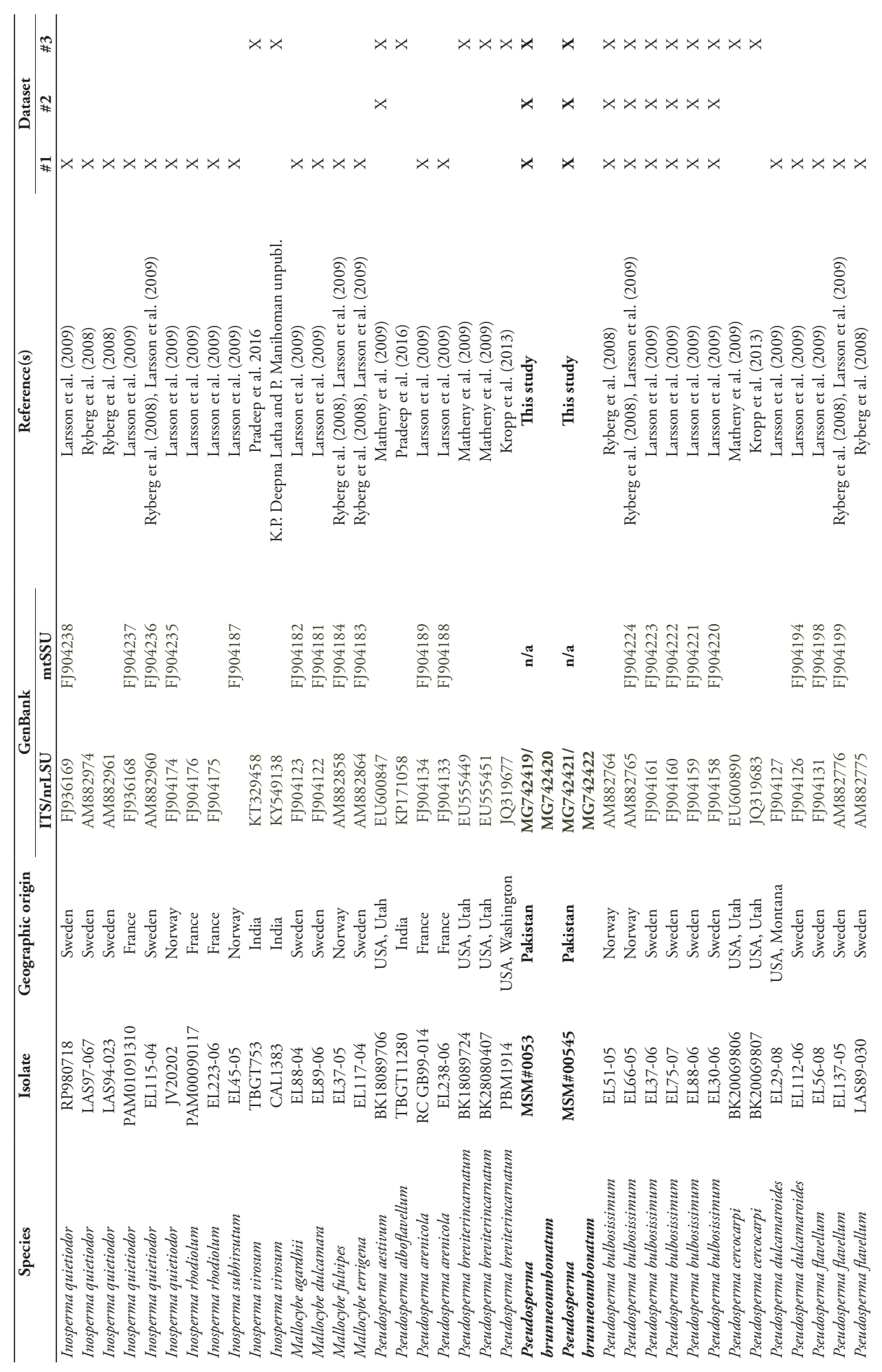




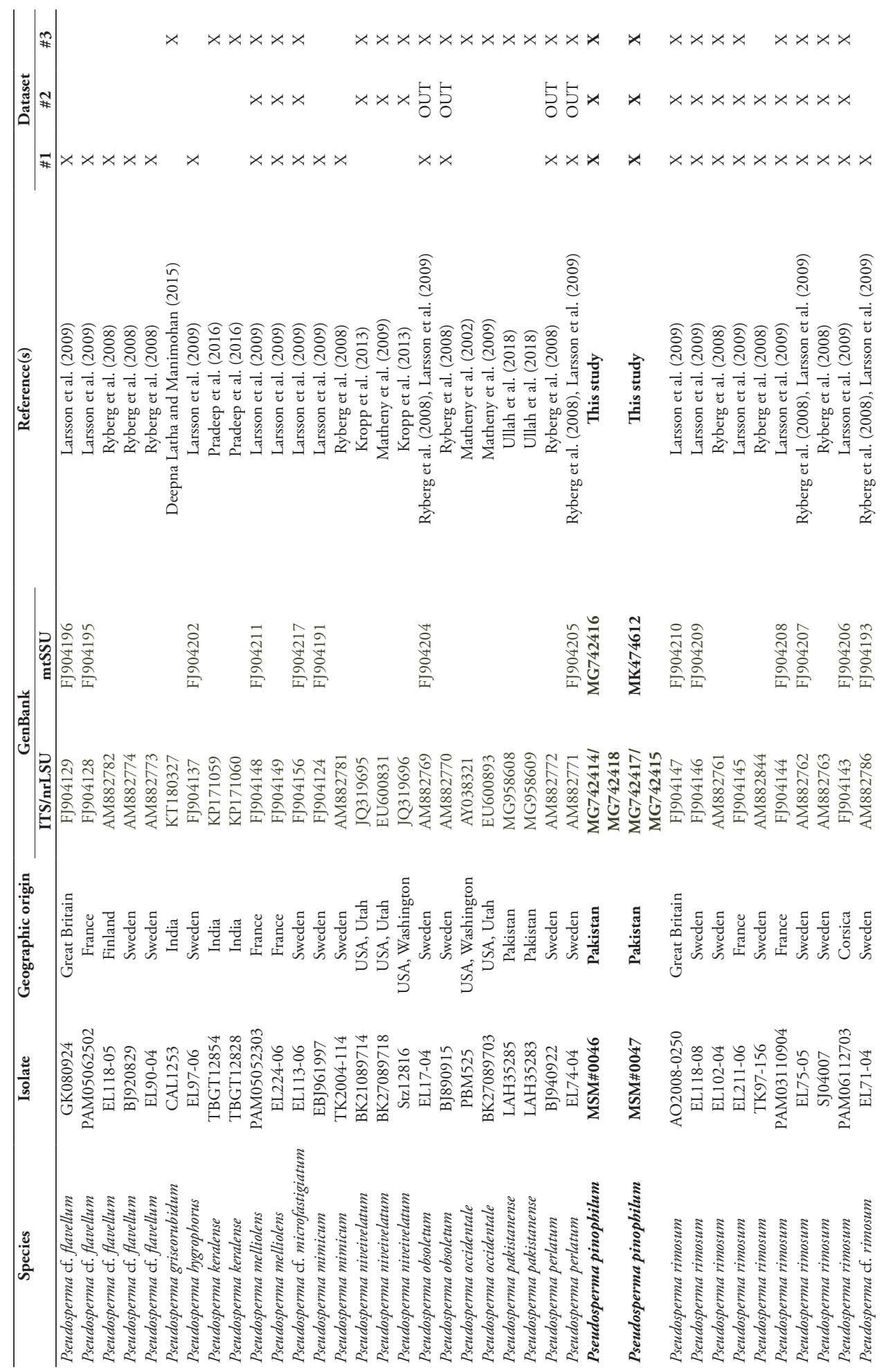




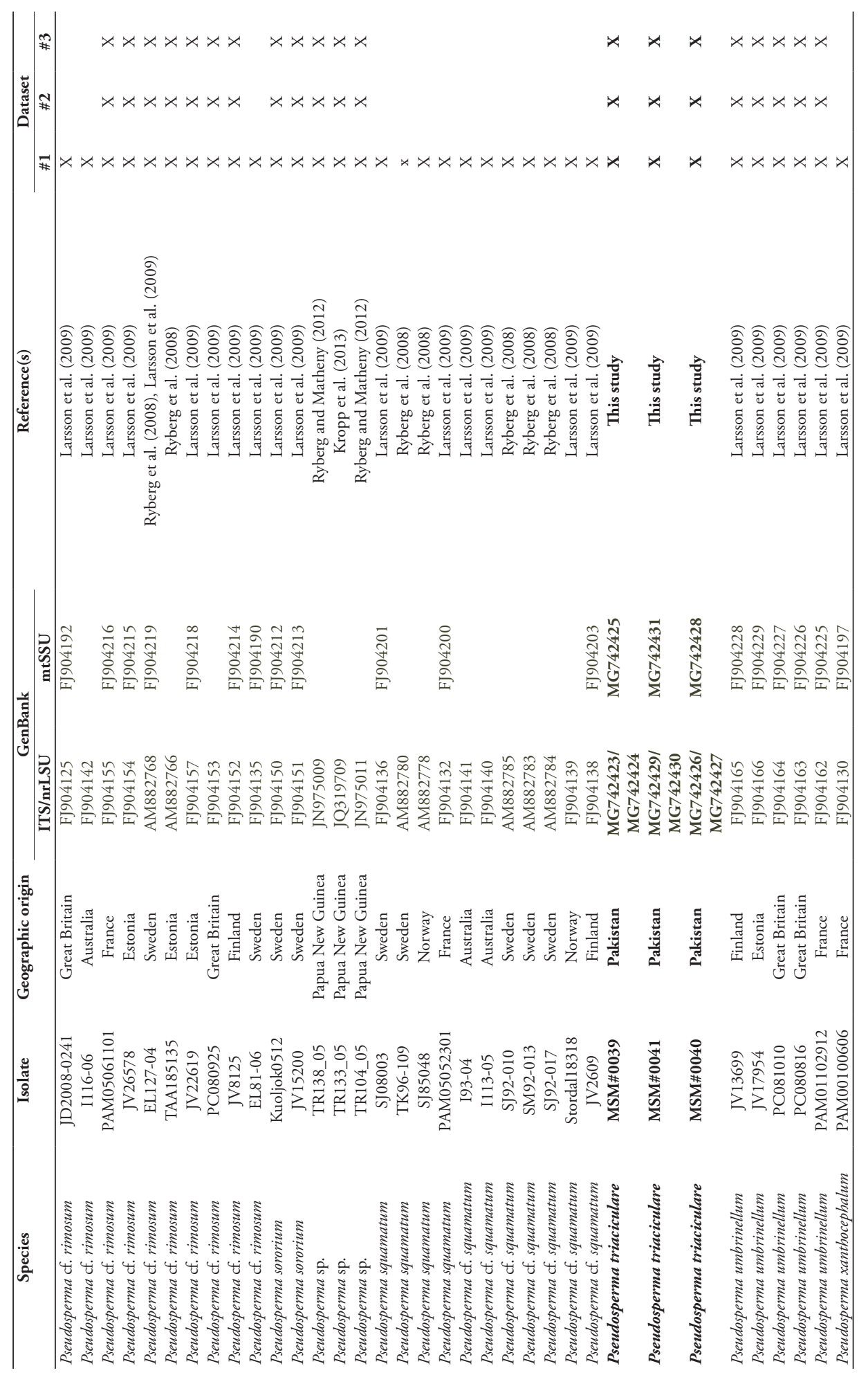




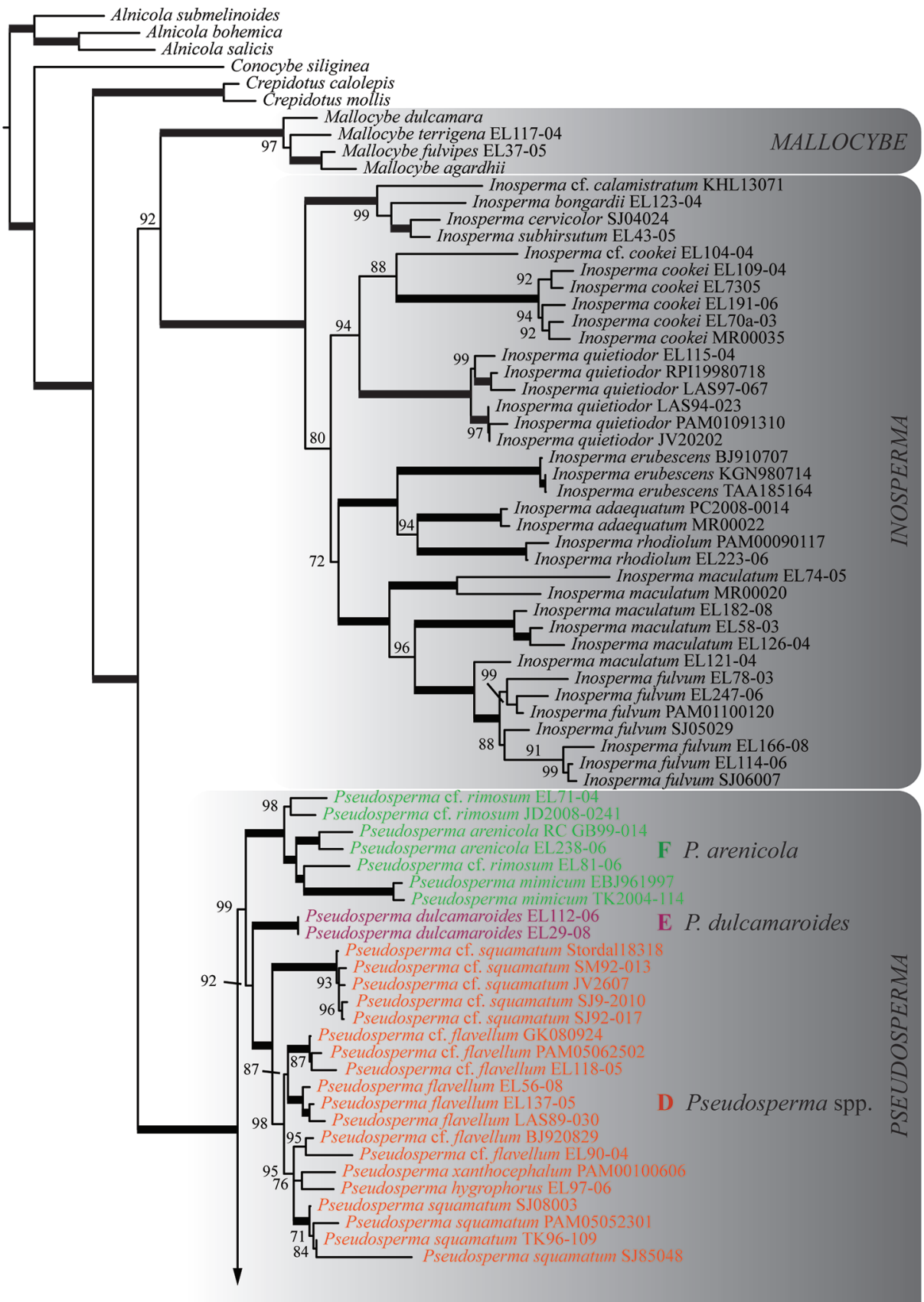

Figure I. The best-scoring ML tree $(-\ln L=27210.474)$ of the Rimosae s.s. and Inosperma clades, reconstructed from the concatenated ITS - nrLSU-mtSSU dataset. ML bootstraps (if $\geq 70$ ) are presented above or in front of the branch leading to each node. Thick branches have maximum support (ML BS = 100). Subclade designations within sect. Rimosae s.s. follow Larsson et al. (2009) in the strict sense. Newlydescribed species are in boldface. 


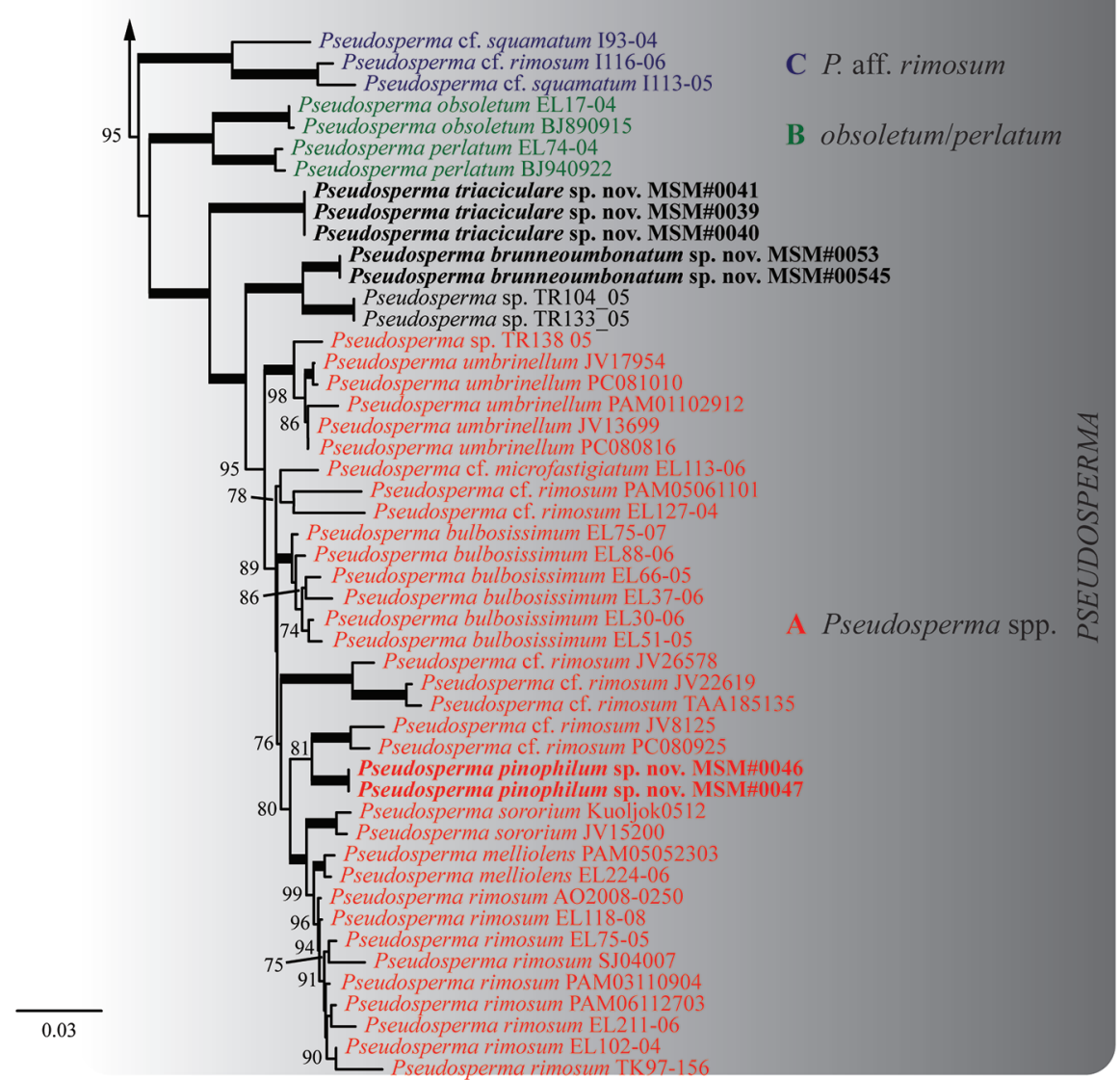

Figure I. Continued.

\section{Taxonomy}

Pseudosperma brunneoumbonatum Saba 8 Khalid, sp. nov.

MycoBank No: 822655

Figure 4

Diagnosis. Characterised by the dark brown umbo and basidiospores I0.3-15.3(-16.7) $\times$ 6.6-9.9 $\mu \mathrm{m}$ and an ecological association with Pinus.

Types. Holotype: Pakistan, Prov. Khyber Pakhtunkhwa, Abbottabad, Shimla, 14 Sep 2012, leg. M. Saba \& A.N. Khalid; MSM\#0053 (LAH 310032); GenBank accession nos. MG742419 (ITS), MG742420 (nrLSU). Paratype: ibid., 6 Aug. 2014; MSM\#00545 (LAH 31003); GenBank accession nos. MG742421 (ITS), MG742422 (nrLSU).

Etymology. From Latin, referring to dark brown colour of the umbo. 
Description. Pileus $20-38 \mathrm{~mm}$ in diam., plane to broadly convex with an acute umbo; margin straight or flaring to deflexed; surface dry, dull, strongly rimose, cracked towards centre but disc smooth and unbroken; strong brown (5YR4/8), disc/umbo deep brown (5YR2/6). Lamellae regular, adnexed to sinuate, close, pale orange yellow (10YR8/4) or pale yellow (5Y9/4), becoming yellowish-brown with age, concolorous with stipe; edges even; lamelullae one tier; edges white and fimbrirate. Stipe 22-40 mm, central to slightly eccentric, equal, recurved squamulose, longitudinally fibrillose, pale yellow (5Y9/4) or light yellowish-brown (10YR7/4), veil not observed. Odour spermatic. Context white, lacking any colour changes where cut or bruised.

Basidiospores 10.3-15.3(-16.7) × 6.6-9.9 $\mu \mathrm{m}[\mathrm{x}=12.5 \times 7.5 \mu \mathrm{m}, \mathrm{Q}=1.2-1.96]$, smooth, phaseoliform or ellipsoid, thin-walled, pale brown to reddish-brown in $\mathrm{KOH}$, apiculus present or absent, apex obtuse. Basidia 27-39 × 10.6-16 $\mu \mathrm{m}$, clavate with refractive contents, primarily 4 -sterigmate, less often 2 -sterigmate, thin-walled, hyaline in KOH; sterigmata 3-6 $\mu \mathrm{m}$ long. Pleurocystidia absent. Cheilocystidia 24-35 × 14-29 $\mu \mathrm{m}$, numerous, clavate, some catenate, hyaline to pale brown, thin-walled. Caulocystidia clavate or cylindrical, similar to cheilocystidia, infrequent. Pileipellis a cutis, hyphae cylindrical, 5-9 $\mu \mathrm{m}$ wide, thin-walled, pale brown in $\mathrm{KOH}$, some with encrustations, septate. Lamellar trama of parallel hyphae, 5-10 $\mu \mathrm{m}$ wide; subhymenium of compact hyphae, 3-6 $\mu \mathrm{m}$ wide. Stipitipellis cylindrical hyphae, hyaline in mass in $\mathrm{KOH}$. All structures inamyloid. Clamp connections present.

Habit and habitat. Occurring in August and September, solitary or in groups, scattered on the forest floor in stands of Pinus roxburghii (Pinaceae).

Notes. In all phylogenetic reconstructions (Figures 1-3), P. brunneoumbonatum sp. nov. is sister to Pseudosperma sp. (isolates TR104_05 and TR133_05). This undescribed species from high-elevations in Papua New Guinea is associated with Castanopsis (Fagaceae). Of the north temperate species, P. brunneoumbonatum is phylogenetically most closely related to $P$. umbrinellum (Figure 3, Table 2). In terms of morphology, P. brunneoumbonatum differs from $P$. umbrinellum by its strong brown pileus with an acute umbo (hazel to cinnamon brown) and somewhat larger basidiospores (measuring 10-13 $\times 5.5-6.5 \mu \mathrm{m}$ in P. umbrinellum). Other related North American taxa are $P$. aestivum (Kropp, Matheny \& Hutchison) Matheny \& Esteve-Rav. and $P$. niveivelatum (D.E. Stuntz ex Kropp, Matheny \& Hutchison) Matheny \& Esteve-Rav. Pseudosperma aestivum can be separated by larger basidiomata and different pileus colouration (yellowish to pale yellow with yellow-brown centre), whereas $P$. niveivelatum has a white stipe and a non-rimose pileus with different colouration (covered with abundant white velipellis) (Kropp et al. 2013). Pseudosperma perlatum (Cooke) Matheny \& EsteveRav. superficially resembles $P$. brunneoumbonatum. However, the slightly larger basidiospores, pale orange yellow stipe and a presumed association with Pinus distinguish the new species from P. perlatum, which is an associate of deciduous trees (Vauras and Huhtinen 1986). It differs from I. rimosum in having broader basidiospores.

Pseudosperma neoumbrinellum (T. Bau \& Y.G. Fan) Matheny \& Esteve-Rav. is an Asian species (described from China) with similar basidioma size and colouration (Bau and Fan 2018). The basidiospores of P. brunneoumbonatum, however, are remarkably larger. 


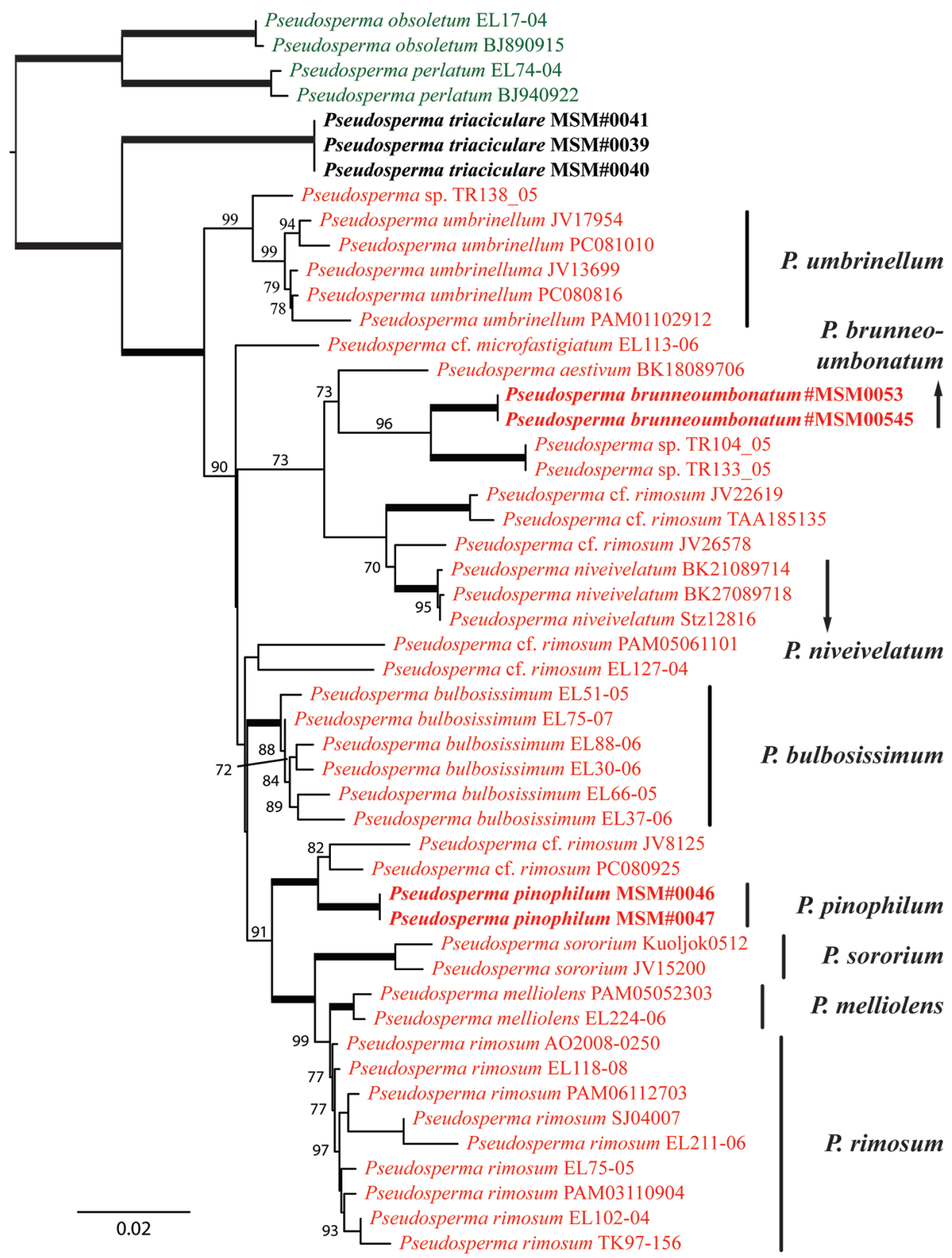

Figure 2. The best-scoring ML tree $(-\operatorname{lnL}=9359.879)$ of Rimosae s.s. subclade $A$, reconstructed from the concatenated ITS-nrLSU-mtSSU dataset. ML bootstraps (if $\geq 70$ ) are presented above or in front of the branch leading to each node. Thick branches have maximum support (ML BS $=100)$. Well-supported clades that represent described species within Rimosae s.s. subclade A are named. Newly-described species are in boldface. 


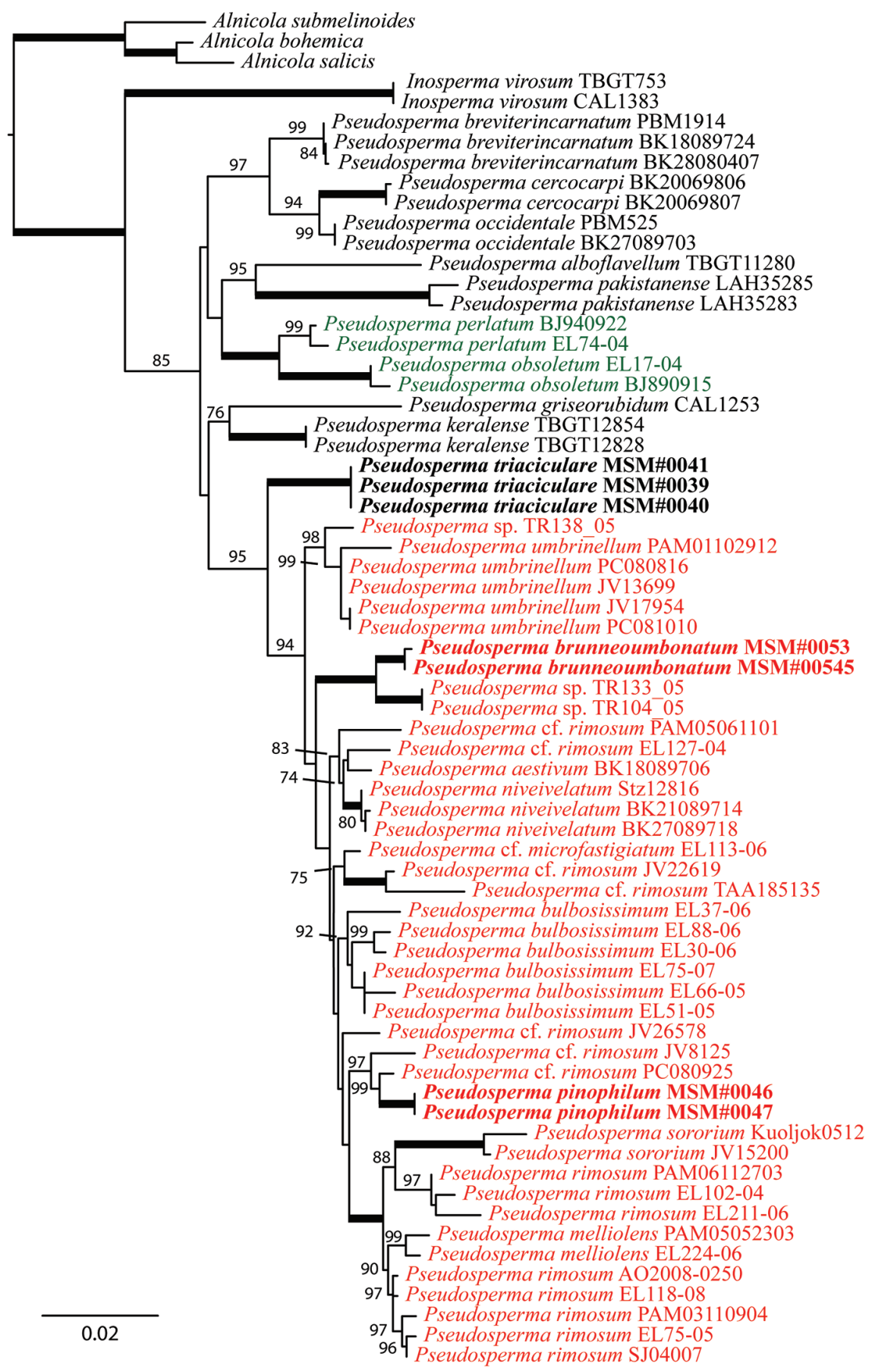

Figure 3. The best-scoring ML tree $(-\operatorname{lnL}=5704.951)$ of Rimosae s.s. subclade A, complemented with recently-described species within sect. Rimosae s.s., reconstructed from the nrLSU dataset. ML bootstraps (if $\geq 70$ ) are presented above or in front of the branch leading to each node. Thick branches have maximum support (ML BS $=100)$. Newly-described species are in boldface. 


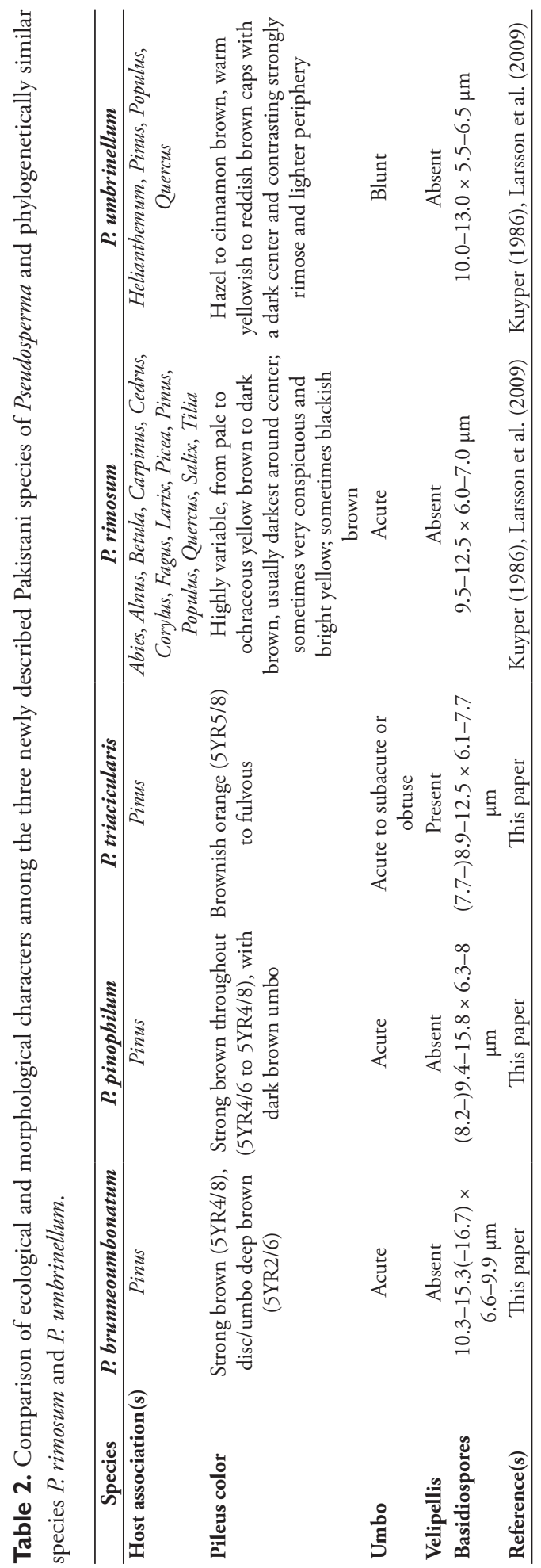


Pseudosperma himalayense (Razaq, Khalid \& Kobayashi) Matheny \& Esteve-Rav. was recently described from Pakistan (Liu et al. 2018) and is similar to P. brunneoumbonatum in having similar pileus size. This species was found at different localities in the western Himalayas, but always near Pinus wallichiana. Pseudosperma himalayense has a much longer stipe (50-80 mm vs. max. $40 \mathrm{~mm}$ in P. brunneoumbonatum); white to pale yellow, olive yellow or light brown pileus; and somewhat smaller basidiospores. Pseudosperma pakistanense (Z. Ullah, S. Jabeen, H. Ahmad \& A.N. Khalid) Matheny \& EsteveRav., another species described from Pakistan, can be differentiated by the presence of pleurocystidia, somewhat smaller basidiospores and phylogenetic placement (Ullah et al. 2018, Figure 3).

The following two species have not yet been recombined in Pseudosperma. However, phylogenetic evidence undoubtedly places both $I$. neglecta E. Horak, Matheny $\&$ Desjardin and I. friabilis Matheny \& Kudzma in the newly-recognised genus Pseudosperma (Horak et al. 2015, Matheny and Kudzma 2019). The new combinations are presented at the end of the taxonomy section. Inocybe neglecta from Thailand was described in the Pseudosperma clade by Horak et al. (2015). While it also lacks pleurocystidia and has a strong brown umbonate pileus, it is different from P. brunneoumbonatum by the smaller pileus (12-18 $\mathrm{mm}$ vs. $20-38 \mathrm{~mm}$ ) and smaller and differently-shaped basidiospores. In addition, I. neglecta is only known from the type locality, growing in a tropical montane forest dominated by Lithocarpus Blume and Castanopsis (D. Don) Spach (both in Fagaceae). Inocybe friabilis, described from North America in the Pseudosperma clade, resembles P. brunneoumbonatum by lacking pleurocystidia and having a similarly coloured pileus. However, I. friabilis has smaller basidiospores, is associated with Quercus and Carya and has an eastern United States distribution.

In The taxonomic studies of the genus Inocybe, Kobayashi (2002) discussed 136 species, of which 13 (including four varieties and three formae) in subgenus Inosperma section Rimosae. These are [all referred to as Inocybe in Kobayashi (2002)]: Inosperma adaequatum (Britzelm.) Matheny \& Esteve-Rav., I. aureostipes (Kobayasi) Matheny \& Esteve-Rav., I. cookei (Bres.) Matheny \& Esteve-Rav., I. erubescens (A. Blytt) Matheny \& Esteve-Rav. [as its synonym I. patouillardii Bres.], I. maculatum (Boud.) Matheny \& Esteve-Rav., Pseudosperma avellaneum (Kobayasi) Matheny \& Esteve-Rav., P. bisporum (Hongo) Matheny \& Esteve-Rav., P. flavellum (P. Karst.) Matheny \& Esteve-Rav., P. macrospermum (Hongo) Matheny \& Esteve-Rav., P. rimosum [as its synonym Inocybe fastigiata (Schaeff.) Quél.], P. squamatum (J.E. Lange) Matheny \& Esteve-Rav., P. transiens (Takah. Kobay.) Matheny \& Esteve-Rav. and P. umbrinellum. Since no sequence data are available for $P$. avellaneum, $P$. bisporum, $P$. macrospermum and $P$. transiens, we will compare their morphology with the newly-proposed Pakistani species.

Pseudosperma avellaneum has a pale greyish ochraceous pileus, its basidiospores are smaller and its cheilocystidia are distinctly narrower (width 9.5-14.5 vs. 14-29 $\mu \mathrm{m}$ ) compared to P. brunneoumbonatum. As the only species in sect. Rimosae (sensu Kobayashi 2002), P. bisporum is 2 -sterigmate. In addition, this species has a generally shorter stipe (17-26 vs. $22-40 \mathrm{~mm}$ in P. brunneoumbonatum), the edges of its lamel- 


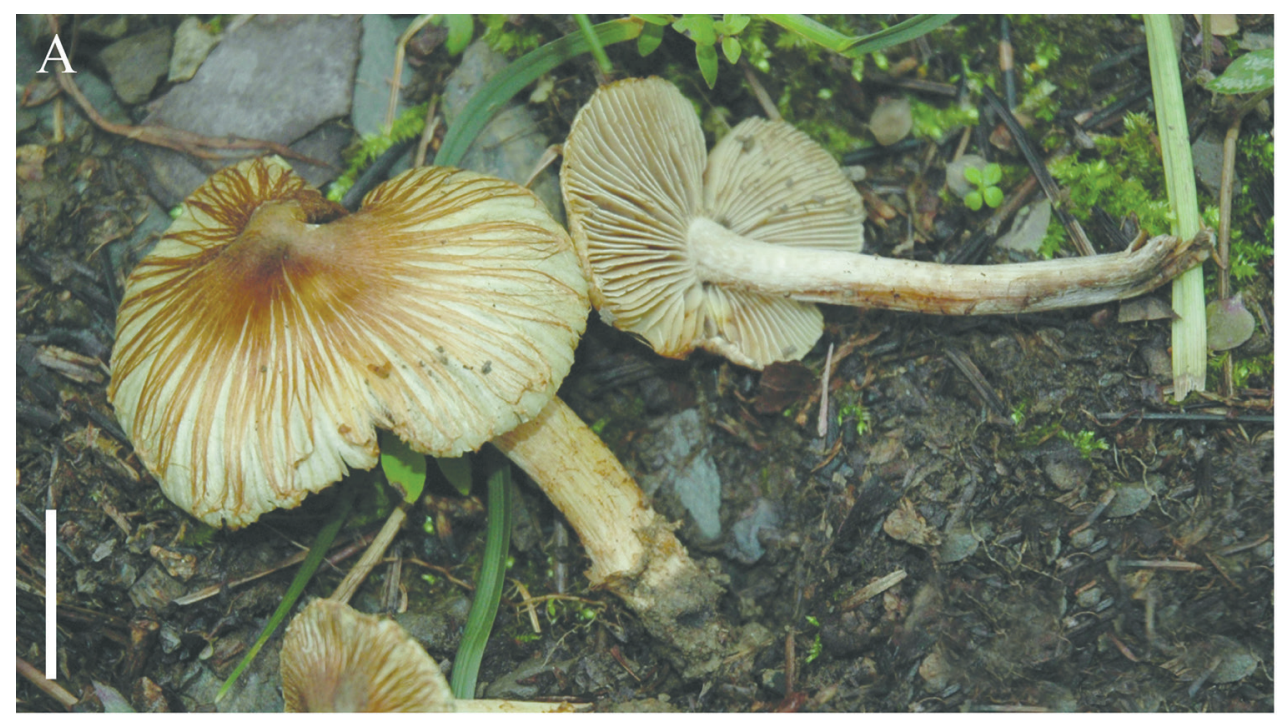

$\mathrm{B}$

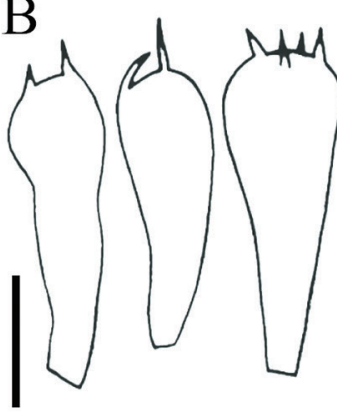

$\mathrm{C}$
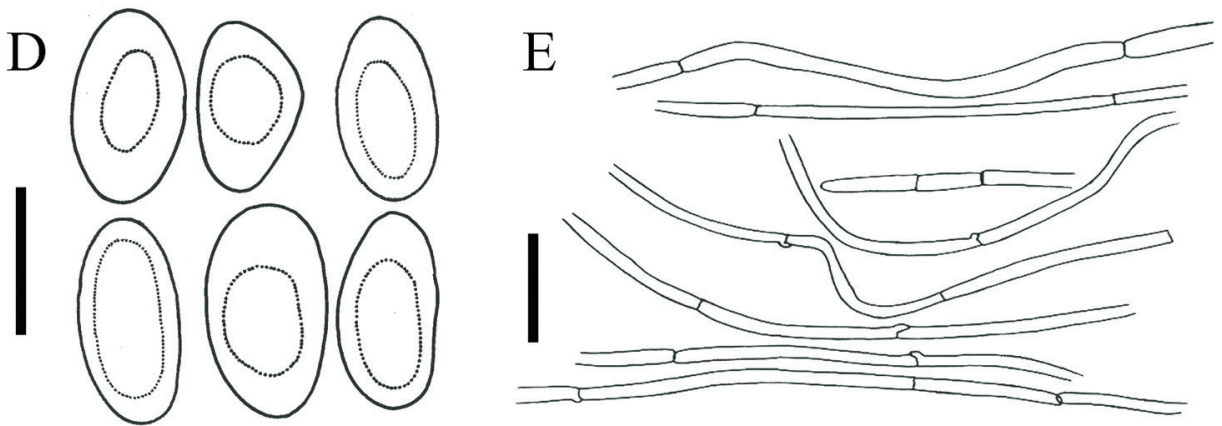

Figure 4. Pseudosperma brunneoumbonatum: A Basidiomata of holotype collection (LAH 310032) B-E microscopic characters: B basidia C cheilocystidia D basidiospores $\mathbf{E}$ pileipellis. Scale bars: $1 \mathrm{~cm}$ (A), $10 \mu \mathrm{m}(\mathbf{B}), 30 \mu \mathrm{m}(\mathbf{C}, \mathbf{E}), 20 \mu \mathrm{m}(\mathbf{D})$.

lae are serrate (with small teeth as a saw) and, again, the cheilocystidia are narrower (width 10.0-13.8 vs. 14-29 $\mu \mathrm{m}$ in P. brunneoumbonatum). Another Japanese species, P. macrospermum, is morphologically different in the following characters: the stipe has 
a bulbous base, the basidia are shorter and narrower and its pileus is much smaller in diameter. Finally, $P$. transiens has a much longer stipe, its basidia are always narrower (up to $9.5 \mu \mathrm{m}$ wide) and its cheilocystidia are both longer and narrower ((29-)38-52 $\times$ 9.5-13.8 $\mu \mathrm{m}$ ) compared to P. brunneoumbonatum.

\section{Pseudosperma pinophilum Saba \& Khalid, sp. nov. \\ MycoBank No: 822656}

Figure 5

Diagnosis. Characterised by the pale to light yellow equal stipe, basidiospores (8.2-)9.4$15.8 \times 6.3-8 \mu \mathrm{m}$ and an ecological association with Pinus.

Types. Holotype: Pakistan, Prov. Khyber Pakhtunkhwa, Abbottabad, Shimla, 14 Sep 2012, leg. M. Saba \& A.N. Khalid; MSM\#0046 (FH 00304582); GenBank accession nos. MG742414 (ITS), MG742418 (nrLSU), MG742416 (mtSSU). Paratype: Pakistan, Prov. Khyber Pakhtunkhwa, Shangla, Yakh Tangay, under Pinus wallichiana, 2 Sep 2013, leg. M. Saba \& A.N. Khalid; MSM\#0047 (LAH 310049); GenBank accession nos. MG742417 (ITS), MG742415 (nrLSU), MK474612 (mtSSU).

Etymology. From Greek, referring to an association with pine species.

Description. Pileus 16-31 mm in diam., convex, broadly convex or plane with an acute umbo; margin straight or flaring to deflexed; surface dry, dull, rimose, cracked towards centre, strong brown throughout (5YR4/6 to 5YR4/8) with dark brown umbo. Lamellae regular, adnexed to sinuate, close, white when young, light olivaceous at maturity; edges even. Stipe 54-70 mm, central, equal, longitudinally fibrillose, white with pale greenish-yellow (10Y9/4) or light yellow (5Y9/6) tinge or olivaceous tinge; veil not observed. Context white. Odour not distinctive.

Basidiospores (8.2-)9.4-15.8 $\times 6.3-8.0 \mu \mathrm{m}[\mathrm{x}=13.5 \times 7.6 \mu \mathrm{m}, \mathrm{Q}=1.4-1.9]$, smooth, phaseoliform or ellipsoid, thin-walled, pale brown to golden brown in $\mathrm{KOH}$, apiculus small and not distinctive, apex obutse. Basidia 21-40 × (9-) 11-14 $\mu \mathrm{m}$, clavate with refractive contents, primarily 4-sterigmate, less often 2-sterigmate, thin-walled, hyaline in $\mathrm{KOH}$; sterigmata 2.5-4.0 $\mu \mathrm{m}$ long. Pleurocystidia absent. Cheilocystidia 25-47 $\times 10-20 \mu \mathrm{m}$, numerous, clavate or cylindrical, hyaline to pale brown in $\mathrm{KOH}$, thin-walled. Caulocystidia not observed. Pileipellis a cutis of repent hyphae, hyphae cylindrical, 4-12 $\mu \mathrm{m}$ wide, thin-walled, pale brown in $\mathrm{KOH}$, septate. Lamellar trama of parallel hyphae, 5-11 $\mu \mathrm{m}$ wide; subhymenium of compact hyphae, 3-6 $\mu \mathrm{m}$ wide. Stipitipellis cylindrical hyphae, $5-12 \mu \mathrm{m}$ wide, hyaline in mass in $\mathrm{KOH}$; all structures inamyloid. Clamp connections present.

Habit and habitat. Occurring in September, solitary or in groups, scattered on the forest floor in stands of Pinus roxburghii and P. wallichiana (Pinaceae).

Notes. Both P. brunneoumbonatum and P. pinophilum are placed in sect. Rimosae s.s. subclade A (Figures 1-3), which corresponds to P. rimosum senso lato, including the several formae and variations described for this species (Larsson et al. 2009). Pseudosperma pinophilum clusters with $P$. cf. rimosum (isolates JV1825 and PC080925). The pale yellow 

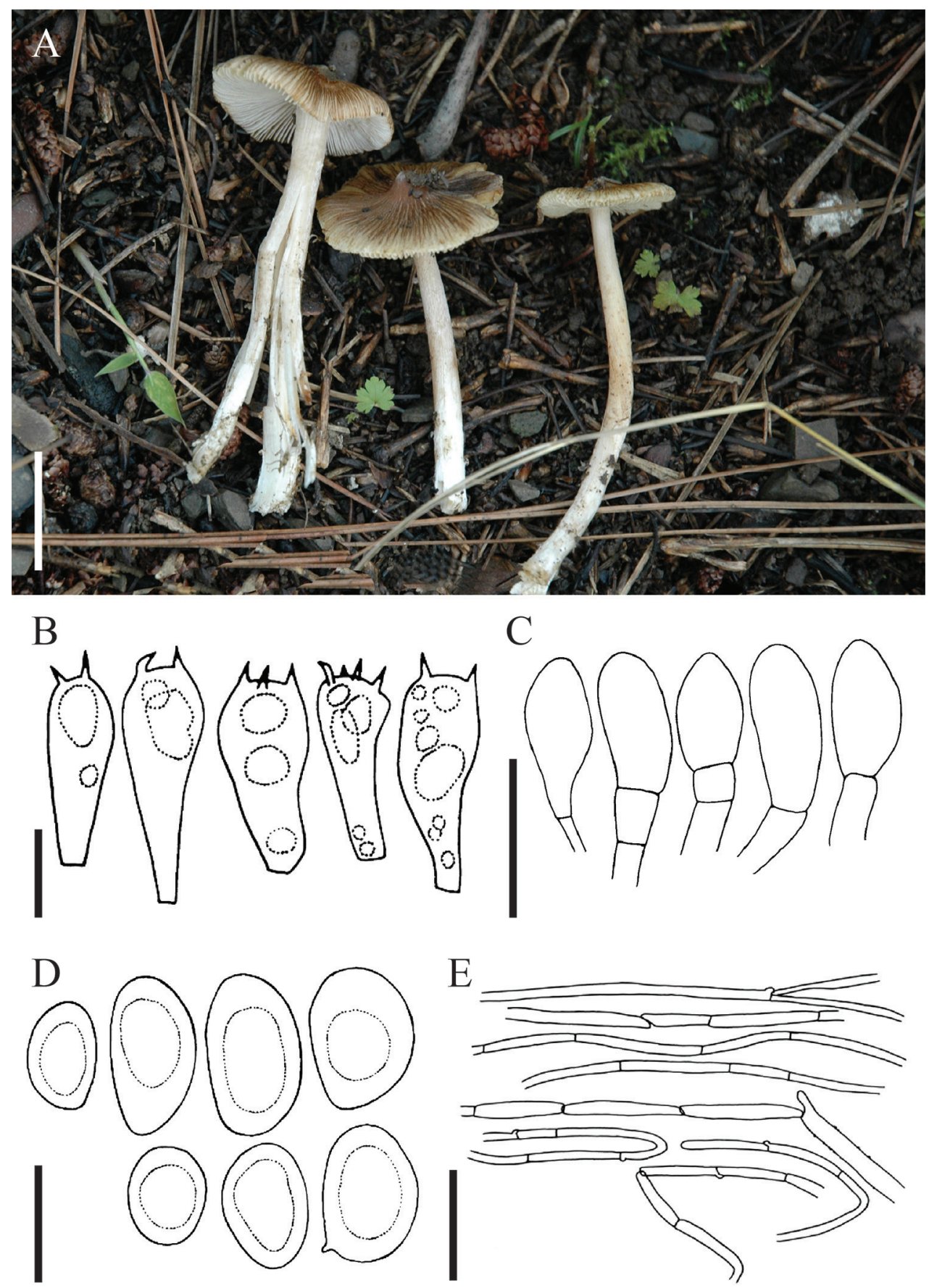

Figure 5. Pseudosperma pinophilum: A Basidiomata of holotype collection (FH 00304582) B-E microscopic characters: $\mathbf{B}$ basidia $\mathbf{C}$ cheilocystidia $\mathbf{D}$ basidiospores $\mathbf{E}$ pileipellis. Scale bars: $1 \mathrm{~cm}(\mathbf{A}), 10 \mu \mathrm{m}(\mathbf{B}$, D), $30 \mu \mathrm{m}(\mathbf{C}, \mathbf{E})$. 
to light yellow tinged, equal stipe in $P$. pinophilum is very different compared to the white (rarely tinged with ochre), sub-bulbous stipe typical for $P$. rimosum. Moreover, $P$. pinophilum has broader basidiospores $((8.2-) 9.4-15.8 \times 6.3-8.0 \mu \mathrm{m})$ compared to $P$. rimosum $(9-11(-13) \times 4.5-6.0 \mu \mathrm{m})$. Also P. brunneoumbonatum has broader - and generally larger - basidiospores $(10.3-15.3(-16.7) \times 6.6-9.9 \mu \mathrm{m})$ compared to $P$. rimosum. Pseudosperma sororium is relatively closely related to $P$. pinophilum and can be differentiated in having different pileus colouration (greyish-brown to pinkish-grey or pale pinkish-beige) and measurement of basidiospores $(10-12.5 \times 5.5-6.0 \mu \mathrm{m})$ (Kauffman 1926).

Two more species of Pseudosperma are known from Pakistan; both $P$. himalayense and P. pakistanense were described, based on material collected in Pakistan. Pseudosperma himalayense was found near Pinus wallichiana trees, but an ITS sequence generated from root tips (GenBank acc. no. HG796995) confirmed an ectomycorrhizal association with Quercus incana (Liu et al. 2018). It can be distinguished from P. pinophilum by the pale yellowish to camel brown, fibrillose pileus; longer cheilocystidia (43-60 $\mu \mathrm{m}$ vs. 25-47 $\mu \mathrm{m}$ ); and much thicker pileipellis. In addition, P. himalayense was resolved as sister to $P$. cf. microfastigiatum (Kühner) Matheny \& Esteve-Rav. in Liu et al.'s (2018) ITS phylogeny. Pseudosperma pakistanense was found in a mixed conifer-dominated forest with some deciduous trees, under Quercus incana (Ullah et al. 2018). This species can be differentiated from the new species by the presence of pleurocystidia, the smaller stipe (50 $\mathrm{mm}$ vs. $54-70 \mathrm{~mm}$ in P. pinophilum) and its phylogenetic position (Ullah et al. 2018). In our nrLSU phylogeny, P. pakistanense was retrieved as sister to $P$. alboflavellum (C.K. Pradeep \& Matheny) Haelew. (Figure 3).

The Japanese species in sect. Rimosae without sequence data from Kobayashi (2002), P. avellaneum, P. bisporum, P. macrospermum and P. transiens, are also different from $P$. pinophilum in their morphology. Pseudosperma avellaneum has smaller basidiospores and the pileipellis hyphae are almost hyaline (vs. pale brown in P. pinophilum). Pseudosperma bisporum has lamellae with serrate edges, its stipe is much shorter (17-26 vs. $54-70 \mathrm{~mm}$ in $P$. pinophilum), the basidia are 2-sterigmate, the cheilocystidia are usually shorter (max. $31 \mu \mathrm{m}$ in length) and the pileipellis hyphae are smaller in diameter. Pseudosperma macrospermum has a smaller pileus diameter, a shorter stipe, narrower basidia, usually shorter cheilocystidia and pileipellis hyphae that are smaller in diameter. Finally, both the basidiospores (4.8-6.5 vs. 6.3-8.0 $\mu \mathrm{m}$ in P. pinophilum) and basidia (8.8-9.5 vs. (9-)11-14 $\mu \mathrm{m}$ in P. pinophilum) of $P$. transiens are narrower. In addition, the cheilocystidia of $P$. pinophilum are hyaline to pale brown in $\mathrm{KOH}$, whereas in P. transiens, they are "rarely filled with yellowish brown contents" (Kobayashi 2002).

\section{Pseudosperma triaciculare Saba \& Khalid, sp. nov. \\ MycoBank No: 822657}

Figure 6

Diagnosis. Characterised by the acutely umbonate brownish-orange to fulvous pileus, the presence of a pale velipellis coating on the pileus, septate cheilocystidia and an ecological association with Pinus. 
Types. Holotype: Pakistan, Prov. Khyber Pakhtunkhwa, Mansehra, Batrasi, under Pinus roxburghii, 3 Aug 2014, leg. M. Saba \& A.N. Khalid; MSM\#0039 (LAH 310054); GenBank accession nos. MG742423 (ITS), MG742424 (nrLSU), MG742425 (mtSSU). Paratypes: ibid., 3 Aug 2014; MSM\#0040 (LAH 310055); GenBank accession nos. MG742426 (ITS), MG742427 (nrLSU), MG742428 (mtSSU). Ibid., 3 Aug 2014; MSM\#0041 (LAH 310056); GenBank accession nos. MG742429 (ITS), MG742430 (nrLSU), MG742431 (mtSSU). Pakistan, Prov. Khyber Pakhtunkhwa, Abbottabad, Shimla, 14 Sep 2012, leg. M. Saba \& A.N. Khalid; MSM\#0038 (FH 00304561).

Etymology. From Latin, meaning "three-needled," with reference to the association with the three-needled pine Pinus roxburghii.

Description. Pileus I 2-29 mm in diam., conical when young, plane to convex at maturity, with acute to subacute or obtuse umbo; margin radially rimose, straight or flaring to uplifted; surface dry, dull, colour brownish-orange (5YR5/8) to fulvous, presence of a pale velipellis coating over the disc. Lamellae regular, adnexed to sinuate, close, pale orange yellow (10YR8/4), edges even; two tiers of lamelullae. Stipe 19-60 mm, central, equal, fibrillose, white with pale orange yellow tinge (10YR8/4). Odour mild, not diagnostic.

Basidiospores (7.7-) 8.9-12.5 × 6.1-7.7 $\mu \mathrm{m}[\mathrm{x}=10.2 \times 6.9 \mu \mathrm{m}, \mathrm{Q}=1.64-2.2]$, smooth, mostly elliptic, thin-walled, yellowish-brown in $\mathrm{KOH}$, apiculus present small and indistinctive. Basidia 24-36 × (9-)10-13 $\mu \mathrm{m}$, clavate to broadly clavate with refractive contents, 4-sterigmate, thin-walled, hyaline in $\mathrm{KOH}$; sterigmata 2.5-4.0 $\mu \mathrm{m}$ long. Pleurocystidia absent. Cheilocystidia cylindrical to clavate, septate, some with sub-capitate apices, terminal cells $23-54 \times 9-16 \mu \mathrm{m}$, non-encrusted, hyaline, thinwalled. Caulocystidia 36-98 × 7-14 $\mu \mathrm{m}$, cylindrical, non-encrusted, hyphoid, thinwalled. Pileipellis a cutis, hyphae cylindrical, 6-12 $\mu \mathrm{m}$ wide, thin-walled, golden brown or yellowish-brown in $\mathrm{KOH}$, without encrustations, septate. Lamellar trama of parallel hyphae, 6-12 $\mu \mathrm{m}$ wide; subhymenium of compact hyphae, 3-6 $\mu \mathrm{m}$ wide. Stipitipellis cylindrical hyphae, $2-12 \mu \mathrm{m}$ wide, hyaline in mass in $\mathrm{KOH}$; all structures inamyloid. Clamp connections present.

Habit and habitat. Occurring in August to September, solitary or in groups, scattered on the forest floor in stands of Pinus roxburghii (Pinaceae).

Notes. Pseudosperma triaciculare has been found in association with Pinus roxburghii, the three-needled pine. This new species forms a distinct monophyletic group without clear affinities outside of Rimosae s.s. subclade A (Figures 1-3). Some of the unique features of this species are the umbonate brownish-orange to pale orange yellow pileus; cylindrical to clavate cheilocystidia; and cylindrical, non-encrusted, hyphoid caulocystidia. Allied species include P. brunneoumbonatum, P. griseorubidum (K.P.D. Latha \& Manim.) Matheny \& Esteve-Rav., P. keralense [synonym I. rimulosa C.K. Pradeep \& Matheny] and P. umbrinellum. Pseudosperma triaciculare shares the same presumed Pinus association and shape of basidiomata with $P$. brunneoumbonatum, but can be distinguished by its brownish-orange pileus and smaller basidiospores. Pseudosperma umbrinellum is differentiated from $P$. triaciculare by the presence of an obtuse umbo (acute in P. triaciculare), yellowish- or reddish-brown pileus (brownish-orange in P. triaciculare), somewhat narrower basidiospores $(5.5-6.5 \mu \mathrm{m}$ vs. 6.1-7.7 $\mu \mathrm{m})$ and a broad host range, including species in Cistaceae, Fagaceae, Pinaceae and Salicaceae (Larsson et al. 2009). 


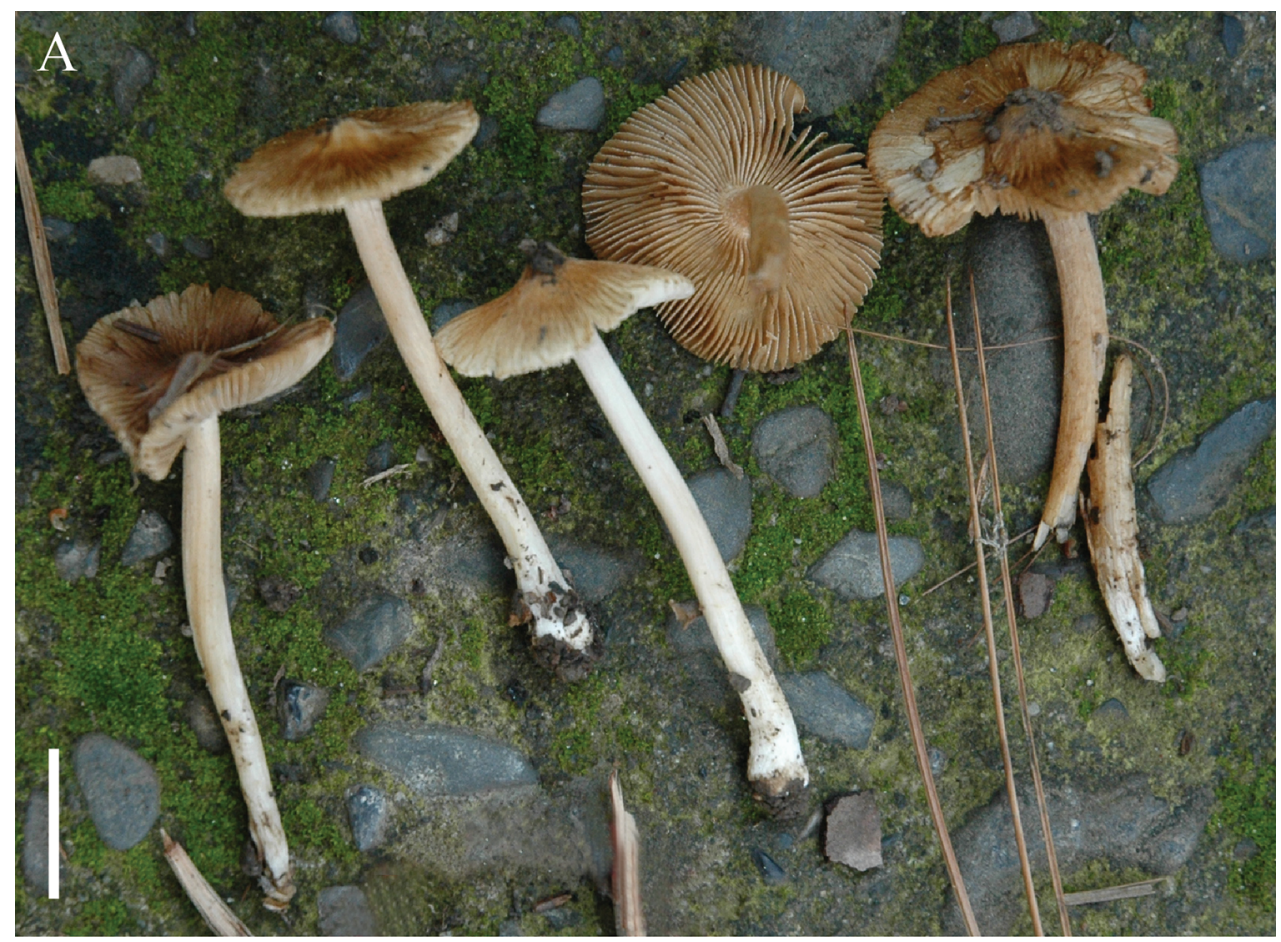

B
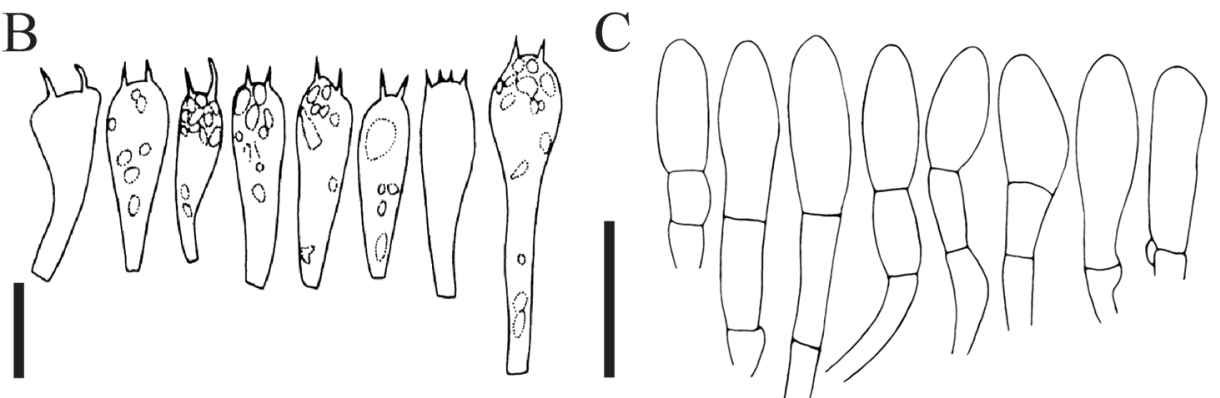

$\mathrm{D}$

$\mathrm{E}$
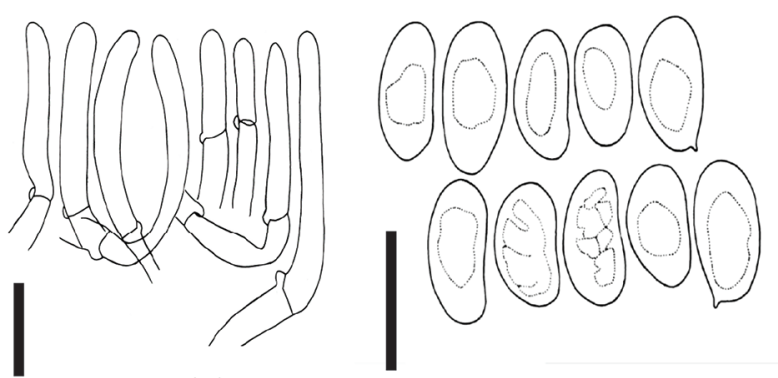

$\mathrm{F}$

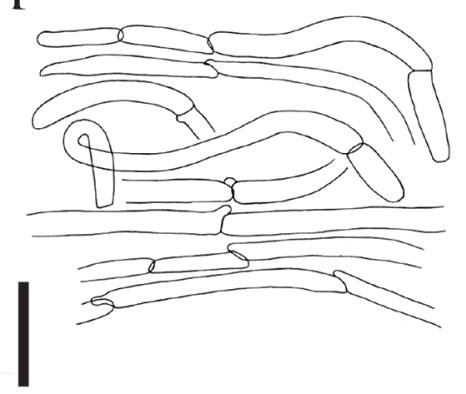

$H$

Figure 6. Pseudosperma triaciculare: A Basidiomata of paratype collection (FH 00304561) B-F microscopic characters: B Basidia $\mathbf{C}$ cheilocystidia $\mathbf{D}$ caulocystidia $\mathbf{E}$ basidiospores $\mathbf{F}$ pileipellis. Scale bars: $1 \mathrm{~cm}(\mathbf{A}), 10 \mu \mathrm{m}(\mathbf{B}, \mathbf{E}), 30 \mu \mathrm{m}(\mathbf{C}, \mathbf{D}, \mathbf{F})$. 
Pseudosperma triaciculare is most closely related to $P$. griseorubidum and $P$. keralense, described recently from tropical India (Latha and Manimohan 2015, Pradeep et al. 2016, Figure 3). Pseudosperma griseorubidum can be differentiated by its pileus, which is greyishred and rarely with an umbo. In addition, $P$. griseorubidum is associated with members of Dipterocarpaceae (Latha and Manimohan 2015). The differences between P. keralense and P. triaciculare are more subtle. Pseudosperma keralense can be separated based on the following features: its lamellae have serrate edges and its basidiospores are narrower on average (6.1 vs. $6.9 \mu \mathrm{m}$ in P. triaciculare). It is also phylogenetically clearly different; the ITS sequence of the holotype collection (GenBank acc. no. KM924523) is $84.11 \%$ identical to the holotype of P. triaciculare, whereas the LSU (KM924518) is $95.13 \%$ identical.

Other similar Asian species include P. himalayense, P. neoumbrinellum, P. pakistanense and P. yunnanense (T. Bau \& Y.G. Fan) Matheny \& Esteve-Rav. Pseudosperma triaciculare resembles $P$. neoumbrinellum in its pileus and basidiospores. However, it is easily differentiated by the characteristic brownish-orange to fulvous colouration of its pileus, whereas the pileus of $P$. neoumbrinellum is chocolate to dark brown in colour (Bau and Fan 2018). In addition, the shape and size of caulocystidia in these two species are very different: $20-48 \times 10-17 \mu \mathrm{m}$ in $P$. neoumbrinellum vs. $36-98 \times 7-14 \mu \mathrm{m}$ in $P$. triaciculare. Pseudosperma triaciculare is different from the recently-described $P$. himalayense from Pakistan (Liu et al. 2018) by the presence of a velipellis and a shorter stipe (16-60 vs. 50-80 $\mu \mathrm{m})$. Pseudosperma pakistanense is separated from P. triaciculare by the absence of velipellar hyphae (unless the authors referred to the velipellis by their description of "[pileus] sometimes peeling off in the form of fine threads"), presence of pleurocystidia and a generally wider stipitipellis lacking caulocystidia (Ullah et al. 2018). Finally, P. yunnanense, described from China, also has velipellar hyphae, but its basidiomata are much larger in size (pileus $30-60 \mathrm{~mm}$ in diam., stipe $60-70 \mathrm{~mm}$ ) and it lacks caulocystidia (Bau and Fan 2018). We did not include P. yunnanense in our phylogenetic analyses, but blasted the ITS sequence of the holotype collection (GenBank acc. no. MH047250) against $P$. triaciculare, resulting in $89.09 \%$ identity. Pseudosperma yunnanense is phylogenetically most similar to $P$. perlatum.

Finally, P. avellaneum, P. bisporum, P. macrospermum and $P$. transiens from Kobayashi's (2002) morphological Inocybe treatment are all different from $P$. triaciculare. Of all four, $P$. avellaneum is probably most difficult to separate from the new species: its pileus is pale greyish-ochraceous, the stipe is less slender and - this seems the best character for separating both species - no caulocystidia were observed. Pseudosperma bisporum has lamellae with serrate edges, 2-sterigmate basidia and pileipellis hyphae that are smaller in diameter. In addition, again, no caulocystidia were observed in this species. Compared to $P$. triaciculare, the basidiospores of $P$. macrospermum are longer (10.5-)14.0-15.5(-18.3) vs. (7.7-)8.9-12.5) $\mu \mathrm{m}$, its basidia are narrower (8.8-9.5($12.5)$ vs. (9-) 10-13 $\mu \mathrm{m})$ and its cheilocystidia are wider (16-18 vs. 9-16 $\mu \mathrm{m})$. Pseudosperma transiens has basidiospores (4.8-6.5 vs. 6.1-7.7 $\mu \mathrm{m}$ ) and basidia (8.8-9.5 vs. (9-)10-13 $\mu \mathrm{m}$ ) that are both narrower than those in P. triaciculare. In addition, the pileus of $P$. transiens is coloured brown to dark brown, whereas $P$. triaciculare has a brownish-orange to fulvous pileus. 


\section{New combinations}

During our studies of Inocybe sensu lato, we came across species of Inocybe that had not been recombined in the appropriate genera after Matheny et al. (2019) proposed a new generic system. Five names are recombined in Inosperma, Mallocybe and Pseudosperma.

Inosperma vinaceobrunneum (Matheny, Ovrebo \& Kudzma) Haelew., Index Fungorum 436: 1 (2020).

Index Fungorum No: IF557431

EInocybe vinaceobrunnea Matheny, Matheny and Kudzma, J. Torrey Bot. Soc. 146(3): 227 (2019). [Basionym]

Note. This combination was made, based on a four-locus phylogeny (ITS, nrLSU, $\mathrm{rpb} 1, \mathrm{rpb} 2)$. Inosperma vinaceobrunneum was retrieved in a clade with two other species (I. rodiolum (Bres.) Matheny \& Esteve-Rav. and an undescribed species), sister to I. adaequatum (Matheny and Kudzma 2019).

Mallocybe erratum (E. Horak, Matheny \& Desjardin) Haelew., comb. nov. Index Fungorum No: IF557512

三 Inocybe errata E. Horak, Matheny \& Desjardin, Phytotaxa 230(3): 210 (2015). [Basionym]

Note. This combination is based on phylogenetic evidence of the holotype (Horak et al. 2015). Based on both nrLSU-alone and nrLSU-rpb1-rpb2 datasets, it is placed deep in Mallocybe. It is highly supported as a sister species to an undescribed Zambia species ("I. microdulcamara" nom. prov.), both sister to M. heimii (Bon) Matheny \& Esteve-Rav. (Matheny et al. 2009, Horak et al. 2015).

\section{Pseudosperma alboflavellum (C.K. Pradeep \& Matheny) Haelew., Index Fungo- rum 436: 1 (2020).}

Index Fungorum No: IF557432

三 Inocybe alboflavella C.K. Pradeep \& Matheny, Pradeep et al., Mycol. Progr. 15: 13 (2016). [Basionym]

Note. This combination was made, based on phylogenetic placement of the isotype (Pradeep et al. 2016, this study). In our nrLSU phylogeny, it was retrieved as a sister species to P. pakistanense with high support (Figure 3). 
Pseudosperma friabile (Matheny \& Kudzma) Haelew., Index Fungorum 436: 1 (2020). Index Fungorum No: IF557433

EInocybe friabilis Matheny \& Kudzma, J. Torrey Bot. Soc. 146(3): 226 (2019). [Basionym]

Note. This combination was made, based on phylogenetic evidence. Pseudosperma friabile is most closely related to P. gracilissimum (Matheny \& Bougher) Matheny \& Esteve-Rav. and P. keralense (K.P.D. Latha \& Manim.) Matheny \& Esteve-Rav., deep in the Pseudosperma clade (fide Matheny 2005, Matheny and Kudzma 2019).

\section{Pseudosperma neglectum (E. Horak, Matheny \& Desjardin) Haelew., comb. nov.} Index Fungorum No: IF557513

三 Inocybe neglecta E. Horak, Matheny \& Desjardin, Phytotaxa 230(3): 208 (2015). [Basionym]

Note. The combination of I. neglecta in genus Pseudosperma is made, based on phylogenetic evidence. Horak et al. (2015) presented the phylogenetic reconstruction of an nrLSU dataset and found high statistical support for the Pseudosperma clade (fide Matheny 2005) including P. neglectum. While P. neglectum was retrieved as sister to the remaining members of the Pseudosperma clade, there was no support for this relationship. The same result was also found by Kropp et al. (2013). In addition, blasting the ITS sequence of the holotype (GenBank acc. no. EU600829) against sequences from type materials, resulted in P. occidentale (Kropp, Matheny \& Hutchison) Matheny \& Esteve-Rav. and $P$. illudens (Matheny, Bougher \& G.M. Gates) Matheny \& Esteve-Rav. with the highest percentages of identity $(96.46 \%$ and $96.28 \%$, respectively).

\section{Discussion}

Pakistan is located in southern Asia. This country is geographically diverse, ranging from the mountainous northern part, where the Himalayas meet their westernmost end, to the southern part with the coastal area along the Arabian Sea. Following the KöppenGeiger classification system for climate, 20 types can be found in Pakistan - including four arid, six temperate, eight cold and even two polar (Beck et al. 2018). Note that despite this diversity in climate types, most of the country has a hot desert climate ( $B W h$, Peel et al. 2007). Pakistan has a very rich flora; in an ongoing effort to write the Flora of Pakistan, S.I. Ali and colleagues identified 5,521 plant species in 1,572 genera thus far (Ali 2008). When keeping the ratio between vascular plants and fungi (1:6) in mind (sensu Hawksworth 1991), this number of plants only hints at the true potential of in-depth mycological studies in Pakistan, which has been traditionally under-explored. 
The multiple geographic features, different climates and plant species richness in Pakistan are suggestive of a high diversity of fungal species. In recent years, many papers have been published, describing new species from different fungal groups collected in Pakistan (e.g. Razaq et al. 2012, Nawaz et al. 2013, Thongklang et al. 2014, Qasim et al. 2015a, 2015b, Sarwar et al. 2015, Hussain et al. 2016, 2017, 2018, Jabeen et al. 2016, Farooqi et al. 2017, Naseer et al. 2018, Ullah et al. 2018, Saba et al. 2019a, 2019b, Kiran et al. 2020). Thirty-five species of Inocybe sensu lato are reported from Pakistan (Ahmad et al. 1997, Ilyas et al. 2013, Saba et al. 2015, Jabeen et al. 2016, Farooqi et al. 2017, Razaq and Shahzad 2017, Naseer et al. 2018, Ullah et al. 2018, Song et al. 2019, this study). The genus Pseudosperma is poorly known in Pakistan, with only three species that were known before this study: P. himalayense, P. rimosum and P. pakistanense (Ahmad et al. 1997, Liu et al. 2018, Ullah et al. 2018).

In his dissertation about smooth-spored species of Inocybe from Europe, Kuyper (1986) presented a key to species of sect. Rimosae. He included 12 species [all as Inocybe]: Inosperma adaequatum, I. cookei, I. erubescens, I. maculatum, I. quietiodor (Bon) Matheny \& Esteve-Rav., I. reisneri (Velen.) Matheny \& Esteve-Rav., Pseudosperma arenicola (R. Heim) Matheny \& Esteve-Rav., P. flavellum, P. mimicum (Massee) Matheny \& Esteve-Rav., P. rimosum (sensu lato), P. squamatum and I. vinosistipitatum (Grund \& D.E. Stuntz) Matheny \& Esteve-Rav. Kuyper (1986) followed a conservative approach for $P$. rimosum - citing 31 species and varieties as synonyms and allowing considerable morphological plasticity and broad ecological amplitude. Larsson et al. (2009) followed a less conservative approach and recognised P. obsoletum, P. perlatum and $P$. umbrinellum as separate species in their identification key of Maculata and $R i$ mosae s.s. clades in north-western Europe. These three species were amongst the synonymies of $P$. rimosum as treated by Kuyper (1986). Following both keys, our newly described taxa are most similar to $P$. rimosum and $P$. umbrinellum (Table 2). From our phylogenetic analyses, it is obvious that both $P$. rimosum and $P$. umbrinellum are separated from our Pakistani species. Other, more recently described taxa of Pseudosperma are also differentiated from the newly-proposed species, based on morphology, molecular phylogeny and geographic distribution.

Our phylogenetic analyses revealed that several undescribed species or collections that have not yet been properly identified occur in Rimosae s.s. subclade A (Larsson et al. 2009, Kropp et al. 2012). These are represented by singleton clades and clades including tentatively (cf.) or unidentified isolates. For example, isolates TR104_05 and TR133_05 represent an undescribed species from Papua New Guinea. In addition, isolates JV1825, PC080925, JV22619 and TAA185135 were identified as $P$. cf. rimosum, but represent at least two different species, either undescribed or previously described, but without available DNA sequence data. The isolate JV26578, which forms a singleton clade with unresolved position in our phylogenetic analyses, was also identified as $P$. cf. rimosum, but this identification is again inaccurate. We agree with Larsson et al. (2009) that more taxa need be sampled before the diversity and evolutionary relationships in this section can be fully understood. 


\section{Data availability}

All holotype and paratype collections of the new species are deposited at LAH and $\mathrm{FH}$. The sequences generated during this study are deposited in NCBI GenBank under accession numbers MG742414-MG742431. The sequence alignments generated in the present study are available from figshare (https://doi.org/10.6084/ m9.figshare.c.4701338).

\section{Acknowledgements}

We are highly indebted to the Higher Education Commission (HEC), Islamabad, Pakistan, for funding this project under Phase II, Batch I, Indigenous PhD fellowships programme for 5000 scholars and through the International Research Support Initiative Program (IRSIP). We thank P. Brandon Matheny (University of Tennessee-Knoxville, USA), Olivier Raspé (Botanic Garden Meise, Belgium) and Martin Ryberg (Uppsala University, Sweden) for critically reviewing the manuscript. Finally, we acknowledge the efforts of Meike Piepenbring and Carola Glatthorn (Goethe-Universität Frankfurt, Germany) to provide us with necessary literature during the COVID-19 pandemic and subsequent lockdown.

\section{References}

Ahmad S, Iqbal SH, Khalid AN (1997) Fungi of Pakistan. Sultan Ahmad Mycological Society Pakistan, 1-248.

Ali SI (2008) Significance of flora with special reference to Pakistan. Pakistan Journal of Botany 40(3): 967-971.

Bau T, Fan Y-G (2018) Three new species of Inocybe sect. Rimosae from China. Mycosystema 37: 693-702.

Beck HE, Zimmermann NE, McVicar TR, Vergopolan N, Berg A, Wood EF (2018) Present and future Köppen-Geiger climate classification maps at 1-km resolution. Scientific Data 5: 180214. https://doi.org/10.1038/sdata.2018.214

Bougher NL, Matheny PB, Gates GM (2012) Five new species and records of Inocybe (Agaricales) from temperate and tropical Australia. Nuytsia 22(2): 57-74.

Capella-Gutiérrez S, Silla-Martínez JM, Gabaldón T (2009) TrimAl: a tool for automated alignment trimming in large-scale phylogenetic analyses. Bioinformatics 25: 1972-1973. https://doi.org/10.1093/bioinformatics/btp348

Chernomor O, Von Haeseler A, Minh BQ (2016) Terrace aware data structure for phylogenomic inference from supermatrices. Systematic Biology 65: 997-1008. https://doi. org/10.1093/sysbio/syw037

Darriba D, Taboada GL, Doallo R, Posada D (2012) jModelTest 2: more models, new heuristics and parallel computing. Nature Methods 9(8): 772. https://doi.org/10.1038/nmeth.2109 
Dentinger BT, Didukh MY, Moncalvo JM (2011) Comparing COI and ITS as DNA barcode markers for mushrooms and allies (Agaricomycotina). Plos One 6(9): e25081. https://doi. org/10.1371/journal.pone.0025081

Edgar RC (2004) MUSCLE: multiple sequence alignment with high accuracy and high throughput. Nucleic Acids Research 32: 1792-1797. https://doi.org/10.1093/nar/gkh340

Farooqi A, Aqdus F, Niazi AR, Jabeen S, Khalid AN (2017) Inocybe ahmadii sp. nov. and a new record of I. leptocystis from Pakistan. Mycotaxon, 132(2): 257-269. https://doi. org/10.5248/132.257

Gardes M, Bruns TD (1993) ITS primers with enhanced specificity for basidiomycetes - application to the identification of mycorrhizae and rusts. Molecular Ecology 2(2): 113-118. https://doi.org/10.1080/10635150802429642

Haelewaters D (2020) Nomenclatural novelties. Index Fungorum 436: 1.

Haelewaters D, Dirks AC, Kappler LA, Mitchell JK, Quijada L, Vandegrift R, Buyck B, Pfister DH (2018) A preliminary checklist of fungi at the Boston Harbor islands. Northeastern Naturalist 25(Special Issue 9): 45-76. https://doi.org/10.1656/045.025.s904

Hall TA (1999) BioEdit: A user-friendly biological sequence alignment editor and analysis program for Windows 95/98/NT. Nucleic Acids Symposium Series 41: 95-98.

Hawksworth D (1991) The fungal dimension of biodiversity: magnitude, significance, and conservation. Mycological Research 95(6): 641-655. https://doi.org/10.1016/S09537562(09)80810-1

Hillis DM, Dixon MT (1991) Ribosomal DNA: Molecular evolution and phylogenetic inference. The Quarterly Review of Biology 66: 411-453. https://doi.org/10.1086/417338

Hoang DT, Chernomor O, Von Haeseler A, Minh BQ, Vinh LS (2017) UFBoot2: Improving the ultrafast bootstrap approximation. Molecular Biology and Evolution 35: 518-522. https://doi.org/10.1093/molbev/msx281

Horak E, Matheny PB, Desjardin DE, Soytong K (2015) The genus Inocybe (Inocybaceae, Agaricales, Basidiomycota) in Thailand and Malaysia. Phytotaxa 230(3): 201-238. https:// doi.org/10.11646/phytotaxa.230.3.1

Hussain S, Yousaf N, Afshan NS, Niazi AR, Ahmad H, Khalid AN (2016) Tulostoma ahmadii sp. nov. and T. squamosum from Pakistan. Turkish Journal of Botany 40(2): 218-225. https://doi.org/10.3906/bot-1501-9

Hussain S, Ahmad H, Khalid AN, Niazi AR (2017) Parasola malakandensis sp. nov. (Psathyrellaceae; Basidiomycota) from Malakand, Pakistan. Mycoscience 58(2): 69-76. https://doi. org/10.1016/j.myc.2016.09.002

Ilyas S, Razaq A, Khalid AN (2013) Inocybe nitidiuscula and its ectomycorrhizae with Alnus nitida from Galyat, Pakistan. Mycotaxon 124: 247-254. https://doi.org/10.5248/124.247

Jabeen S, Ahmad I, Rashid A, Khalid AN (2016) Inocybe kohistanensis, a new species from Pakistan. Turkish Journal of Botany 40(3): 312-318. https://doi.org/10.3906/bot-1501-17

Jacobsson S (2008) Key to Inocybe. In: Knudsen H, Vesterholt J (Eds) Funga Nordica. Agaricoid, boletoid and cyphelloid genera. Nordsvamp, Copenhagen, 868-906.

Kauffman CH (1926) The genera Flammula and Paxillus and the status of the American species. American Journal of Botany 13(1): 11-32. https://doi.org/10.1002/j.1537-2197.1926. tb05862.x 
Kiran M, Sattar A, Zamir K, Haelewaters D, Nasir Khalid A (2020) Additions to the genus Chroogomphus (Boletales, Gomphidiaceae) from Pakistan. MycoKeys 66: 23-38. https:// doi.org/10.3897/mycokeys.66.38659

Kobayashi T (2002) The taxonomic studies of the genus Inocybe. Nova Hedwigia 124: 1-246. Kropp BR, Matheny PB, Hutchison LJ (2013) Inocybe section Rimosae in Utah: phylogenetic affinities and new species. Mycologia 105(3): 728-747. https://doi.org/10.3852/12-185

Kuyper TW (1986) A revision of the genus Inocybe in Europe. I. Subgenus Inosperma and the smooth-spored species of subgenus Inocybe. Persoonia Suppl. 3: 1-247.

Kumar S, Stecher G, Tamura K (2016) MEGA7: Molecular Evolutionary Genetics Analysis version 7.0 for bigger datasets. Molecular Biology and Evolution 33(7): 1870-1874. https://doi.org/10.1093/molbev/msw054

Larsson E, Ryberg M, Moreau PA, Mathiesen ÅD, Jacobsson S (2009) Taxonomy and evolutionary relationships within species of section Rimosae (Inocybe) based on ITS, LSU and mtSSU sequence data. Persoonia 23: 86-98. https://doi.org/10.3767/003158509X475913

Latha KPD, Manimohan P (2015) Inocybe griseorubida, a new species of Pseudosperma clade from tropical India. Phytotaxa 221(2): 166-174. https://doi.org/10.11646/phytotaxa.221.2.6

Lee SB, Milgroom MG, Taylor JW (1988) A rapid, high yield mini-prep method for isolation of total genomic DNA from fungi. Fungal Genetics Reports 35(1): 23. https://doi. org/10.4148/19

Liu L-N, Razaq A, Atri NS, Bau T, Belbahri L, Chenari Bouket A, Chen L-P, Deng C, Ilyas S, Khalid AN, Kitaura MJ, Kobayashi T, Li Y, Lorenz AP, Ma Y-H, Malysheva E, Malysheva V, Nuytinck J, Qiao M, Saini MK, Scur MC, Sharma S, Shu L-L, Spirin V, Tanaka Y, Tojo M, Uzuhashi S, Valério-Júnior C, Verbeken A, Verma B, Wu R-H, Xu J-P, Yu Z-F, Zeng H, Zhang B, Banerjee A, Beddiar A, Bordallo JJ, Dafri A, Dima B, Krisai-Greilhuber I, Lorenzini M, Mandal R, Morte A, Nath PS, Papp V, Pavlík J, Rodríguez A, Ševčíková H, Urban A, Voglmayr H, Zapparoli G (2018) Fungal systematics and evolution: FUSE 4. Sydowia 70: 211-286.

Matheny PB (2005) Improving phylogenetic inference of mushrooms with RPB1 and RPB2 nucleotide sequences (Inocybe; Agaricales). Molecular Phylogenetics and Evolution 35(1): 1-20. https://doi.org/10.1016/j.ympev.2004.11.014

Matheny PB (2009) A phylogenetic classification of the Inocybaceae. McIlvainea 18(1): 11-21. Matheny PB, Aime MC, Bougher NL, Buyck B, Desjardin DE, Horak E, Kropp BR, Lodge DJ, Soytong K, Trappe JM, Hibbett DS, Hibbett DS (2009) Out of the Palaeotropics? Historical biogeography and diversification of the cosmopolitan ectomycorrhizal mushroom family Inocybaceae. Journal of Biogeography 36(4): 577-592. https://doi.org/10.1111/ j.1365-2699.2008.02055.x

Matheny PB, Hobbs AM, Esteve-Raventós F (2019) Genera of Inocybaceae: New skin for the old ceremony. Mycologia 112(1): 83-120. https://doi.org/10.1080/00275514.2019.1668906

Matheny PB, Kudzma LV (2019) New species of Inocybe (Inocybaceae) from eastern North America. Journal of the Torrey Botanical Society 146(3): 213-235. https://doi. org/10.3159/TORREY-D-18-00060.1

Matheny PB, Liu YJ, Ammirati JF, Hall BD (2002) Using RPB1 sequences to improve phylogenetic inference among mushrooms (Inocybe, Agaricales). American Journal of Botany 89(4): 688-698. https://doi.org/10.3732/ajb.89.4.688 
Matheny PB, Pradeep CK, Vrinda KB, Varghese SP (2012) Auritella foveata, a new species of Inocybaceae (Agaricales) from tropical India. Kew Bulletin 67(1): 119-125. https://doi. org/10.1007/s12225-012-9329-9

Miller MA, Pfeiffer W, Schwartz T (2010) Creating the CIPRES Science Gateway for inference of large phylogenetic trees. Proceedings of the Gateway Computing Environments Workshop (GCE), 14 Nov 2010, 1-8. https://doi.org/10.1109/GCE.2010.5676129

Munsell Soil Color Charts (1975) Munsell Color Company. Baltimore, Maryland.

Naseer A, Khalid AN, Smith ME (2018) Inocybe shawarensis sp. nov. in the Inosperma clade from Pakistan. Mycotaxon 132(4): 909-918. https://doi.org/10.5248/132.909

Nawaz R, Khalid AN, Hanif M, Razaq A (2013) Lepiota vellingana sp. nov. (Basidiomycota, Agaricales), a new species from Lahore, Pakistan. Mycological Progress 12(4): 727-732. https://doi.org/10.1007/s11557-012-0884-0

Nguyen L-T, Schmidt HA, Von Haeseler A, Minh BQ (2015) IQ-TREE: A fast and effective stochastic algorithm for estimating maximum likelihood phylogenies. Molecular Biology and Evolution 32: 268-274. https://doi.org/10.1093/molbev/msu300

Peel MC, Finlayson BL, McMahon TA (2007) Updated world map of the Köppen-Geiger climate classification, Hydrology and Earth System Sciences 11(5): 1633-1644. https://doi. org/10.5194/hess-11-1633-2007

Pradeep CK, Vrinda KB, Varghese SP, Korotkin HB, Matheny PB (2016) New and noteworthy species of Inocybe (Agaricales) from tropical India. Mycological progress 15(3): 24. https:// doi.org/10.1007/s11557-016-1174-z

Qasim T, Amir T, Nawaz R, Niazi AR, Khalid AN (2015) Leucoagaricus lahorensis, a new species of L. sect. Rubrotincti. Mycotaxon 130(2): 533-541. https://doi.org/10.5248/130.533

Qasim T, Khalid AN, Vellinga EC, Razaq A (2015) Lepiota albogranulosa sp. nov. (Agaricales, Agaricaceae) from Lahore, Pakistan. Mycological Progress 14(5): 24. https://doi. org/10.1007/s11557-015-1037-z

Razaq A, Khalid AN, Vellinga EC (2012) Lepiota himalayensis (Basidiomycota, Agaricales), a new species from Pakistan. Mycotaxon 121: 319-325. https://doi.org/10.5248/121.319

Razaq A, Shahzad S (2017) Additions to the diversity of mushrooms in Gilgit-Baltistan, Pakistan. Pakistan Journal of Botany 49(SI): 305-309.

Ryberg M, Nilsson RH, Kristiansson E, Töpel M, Jacobsson S, Larsson E (2008) Mining metadata from unidentified ITS sequences in GenBank: a case study in Inocybe (Basidiomycota). BMC Evolutionary Biology 8(1): 50. https://doi.org/10.1186/1471-2148-8-50

Saba M, Ahmad I, Khalid AN (2015) New reports of Inocybe from pine forests in Pakistan. Mycotaxon 130(3): 671-681. https://doi.org/10.5248/130.671

Saba M, Haelewaters D, Fiaz M, Khalid AN, Pfister DH (2019a) Amanita mansehraensis, a new species in section Vaginatae from Pakistan. Phytotaxa 409(4): 189-201. https://doi. org/10.11646/phytotaxa.409.4.1

Saba M, Haelewaters, D, Iturriaga T, AshrafT, Khalid AN, Pfister DH (2019b) Geopora ahmadii sp. nov. from Pakistan. Mycotaxon 134(2): 377-389. https://doi.org/10.5248/134.377

Sarwar S, Saba M, Khalid AN, Dentinger BM (2015) Suillus marginielevatus, a new species and S. triacicularis, a new record from Western Himalaya, Pakistan. Phytotaxa 203(2): 169-177. https://doi.org/10.11646/phytotaxa.203.2.6 
Smith AH, Stuntz DE (1950) New or noteworthy fungi from Mt. Rainier National Park. Mycologia 42(1): 80-134. https://doi.org/10.1080/00275514.1950.12017817

Song J, Liang J-F, Mehrabi-Koushki M, Krisai-Greilhuber I, Ali B, Bhatt VK, Cerna-Mendoza A, Chen B, Chen Z-X, Chu H-L, Corazon-Guivin MA, da Silva GA, De Kesel A, Dima B, Dovana F, Farokhinejad R, Ferisin G, Guerrero-Abad JC, Guo T, Han L-H, Ilyas S, Justo A, Khalid AN, Khodadadi-Pourarpanahi S, Li T-H, Liu C, Lorenzini M, Lu J-K, Mumtaz AS, Oehl F, Pan X-Y, Papp V, Qian W, Razaq A, Semwal KC, Tang L-Z, Tian X-L, VallejosTapullima A, van der Merwe NA, Wang S-K, Wang C-Q, Yang R-H, Yu F, Zapparoli G, Zhang M, Antonín V, Aptroot A, Aslan A, Banerjee A, Chatterjee S, Dirks AC, Ebrahimi L, Fotouhifar K-B, Ghosta Y, Kalinina LB, Karahan D, Maiti M, Mookherjee A, Nath PS, Panja B, Saha J, Ševčíková H, Voglmayr H, Yazıcı K, Haelewaters D (2019) Fungal Systematics and Evolution 5. Sydowia 71: 141-245.

Stangl J (1989) Die Gattung Inocybe in Bayern. Hoppea 46: 5-388.

Thongklang N, Nawaz R, Khalid AN, Chen J, Hyde KD, Zhao R, Parra LA, Hanif M, Moinard M, Callac P (2014) Morphological and molecular characterization of three Agaricus species from tropical Asia (Pakistan, Thailand) reveals a new group in section Xanthodermatei. Mycologia 106(6): 1220-1232. https://doi.org/10.3852/14-076

Ullah Z, Jabeen S, Ahmad H, Khalid AN (2018). Inocybe pakistanensis, a new species in section Rimosae s. str. from Pakistan. Phytotaxa 348(4): 279-288. https://doi.org/10.11646/ phytotaxa.348.4.4

Vauras J, Huhtinen S (1986) Finnish records on the genus Inocybe ecology and distribution of four calciphilous species. Karstenia 26(2): 65-72. https://doi.org/10.29203/ka.1986.246

Vilgalys R, Hester M (1990) Rapid genetic identification and mapping of enzymatically amplified ribosomal DNA from several Cryptococcus species. Journal of Bacteriology 172(8): 4238-4246. https://doi.org/10.1128/JB.172.8.4238-4246.1990

White TJ, Bruns TD, Lee SB, Taylor JW (1990) Analysis of phylogenetic relationships by amplification and direct sequencing of ribosomal RNA genes. In: Innis MA, Gelfand DH, Sninsky JJ, White TJ (Eds) PCR Protocols: a guide to methods and applications. Academic Press, San Diego, 315-322. https://doi.org/10.1016/B978-0-12-372180-8.50042-1 